\title{
Mutations in sorghum SBEIIb and SSIla affect alkali spreading value, starch composition, thermal properties and flour viscosity
}

\author{
Stefanie Griebel ${ }^{1} \cdot$ Richard P. Westerman ${ }^{2} \cdot$ Adedayo Adeyanju $^{1}$. Charles Addo-Quaye ${ }^{3}$ - Bruce A. Craig ${ }^{4}$. \\ Clifford F. Weil ${ }^{1}$ - Suzanne M. Cunningham ${ }^{1}$ - Bhavesh Patel ${ }^{5}$ - Osvaldo H. Campanella ${ }^{5,6} \cdot$ Mitchell R. Tuinstra $^{1}$
}

Received: 3 April 2019 / Accepted: 17 September 2019 / Published online: 17 October 2019

(c) The Author(s) 2019

\begin{abstract}
Key message Seven novel alleles of SBEIIb and one allele of SSIIa co-segregated with the ASV phenotype and contributed to distinct starch quality traits important for food-processing applications.

Abstract Sorghum is an important food crop for millions of people in Africa and Asia. Whole-genome re-sequencing of sorghum EMS mutants exhibiting an alkali spreading value (ASV) phenotype revealed candidate SNPs in Sobic.004G163700 and Sobic.010G093400. Comparative genomics identified Sobic.010G093400 as a starch synthase IIa and Sobic.004G163700 as a starch branching enzyme IIb. Segregation analyses showed that mutations in Sobic.010G093400 or Sobic.004G163700 co-segregated with the ASV phenotype. Mutants in SSIIa exhibited no change in amylose content but expressed lower final viscosity and lower starch gelatinization temperature (GT) than starches from non-mutant plants. The sbeIIb mutants exhibited significantly higher amylose levels and starch GT and lower viscosity compared to non-mutant starches and ssIIa mutants. Mutations in SBEIIb had a dosage-dependent effect on amylose content. Double mutants of sbeIIb and ssIIa resembled their sbeIIb parent in amylose content, starch thermal properties and viscosity profiles. These variants will provide opportunities to produce sorghum varieties with modified starch end-use qualities important for the beer brewing and baking industries and specialty foods for humans with diabetes.
\end{abstract}

\section{Introduction}

Communicated by Ian D Godwin.

Electronic supplementary material The online version of this article (https://doi.org/10.1007/s00122-019-03430-0) contains supplementary material, which is available to authorized users.

Mitchell R. Tuinstra

drmitch@purdue.edu; mtuinstr@purdue.edu

1 Department of Agronomy, Purdue University, Lilly Hall of Life Sciences, 915 W State Street, West Lafayette, IN 47907, USA

2 College of Agriculture Administration, Purdue University, West Lafayette, IN 47907, USA

3 Division Natural Sciences and Mathematics, Lewis-Clark State College, Lewiston, ID 83501, USA

4 Department of Statistics, Purdue University, West Lafayette, IN 47907, USA

5 Whistler Carbohydrate Research Center, Purdue University, West Lafayette, IN 47907, USA

6 Department of Food Science and Technology, The Ohio State University, Columbus, OH 43210-1007, USA
Sorghum [Sorghum bicolor (L.) Moench] is important in drought-prone environments (Food and Agriculture Organization of the United Nations 2019a, b; Kimber 2000). It is a staple crop in Africa and Asia and is utilized to produce traditional foods such as stiff porridges and beverages (House et al. 2000). Indigenous to Africa, sorghum flour is less expensive than other imported flours (Taylor and Dewar 2000); thus, it has a great economic value for smallholder farmers.

Starch is a major component of sorghum flour and provides unique texture and nutritional value to foods (BeMiller 2014; BeMiller and Huber 2008; Biliaderis 2009). The two major starch fractions, amylose and amylopectin and their ratio, influence starch functionality (BeMiller and Huber 2008; Jane 2009; Tetlow et al. 2004), where amorphous ( $\alpha-1,6$ branch points) and crystalline (linear $\alpha-1,4$ glucan chains) regions play important roles (Tetlow and Emes 2014). Starch compositions of 70-80\% amylopectin and 15-30\% amylose are reported in normal starches of maize, rice wheat and sorghum (Jane 2009; Biliaderis 2009; 
Shannon et al. 2009; BeMiller and Huber 2008; Waniska and Rooney 2000; Sang et al. 2008). Mutant starches such as waxy can have up to $100 \%$ amylopectin, and high amylose starches can have more than 50\% apparent amylose content (Jane 2009; BeMiller and Huber 2008). Both polymers and their ratio can impact characteristics such as paste viscosity, gel consistency, thickening power and starch GT (BeMiller and Huber 2008; Bouvier and Campanella 2014; Shannon et al. 2009). The melting of the crystalline regions, mainly formed by amylopectin, is critical for starch granule swelling and starch gelatinization (BeMiller and Huber 2008; Bouvier and Campanella 2014). Some of the granule physio-chemical and structural characteristics are directly related to granule swelling and starch pasting properties (Desam et al. 2018) and are positively correlated with the peak viscosity ("the maximum swelling capacity of the starch granules") (Bouvier and Campanella 2014).

Amylose levels in sorghum are positively correlated with final viscosity and setback (increase in viscosity after breakdown) and negatively with breakdown (Hill et al. 2012) and peak viscosity (Beta et al. 2000; Hill et al. 2012). The breakdown is a loss of viscosity of a starch paste and is genotype dependent (Bouvier and Campanella 2014). The viscosity breakdown can be described as the time range after peak viscosity occurred, when the starch granules fall apart due to shear forces while the temperature remains constant (Bouvier and Campanella 2014). The final viscosity develops over time during a temperature reduction in the sample, which is related to the development of starch gel viscosity (Bouvier and Campanella 2014) and consistency. Beta et al. (2000) reported a positive correlation between amylose content and peak starch GT $\left(T_{\mathrm{p}}\right)$ in sorghum (Beta et al. 2000). Thus, end-product quality depends on the functionality of starches, which is influenced by starch characteristics and starch GT (BeMiller and Huber 2008; Biliaderis 2009). Sorghum beer is one example of an important end-product. The high starch GT of sorghum requires higher mashing temperatures, which result in low levels of fermentable sugars (Taylor and Dewar 2000; Taylor 1992). It is therefore desired to develop sorghum genotypes with lower starch GT to increase fermentable sugar output and thus beer quality. Food products containing resistant starches are important end-products consumed by humans with diabetes (Englyst et al. 2007). Resistant starches are correlated with high amylose content (Jane 2009; Tetlow and Emes 2014; Li et al. 2008) and exhibit reduced absorption in humans and demonstrate lower glycaemic index values (Englyst et al. 2007). Amylose extender (ae) is a mutant starch type resulting in high amylose starches in maize ( $\mathrm{Li}$ et al. 2008; Liu et al. 2012a; Nakamura 2015b; Shannon et al. 2009; Tetlow and Emes 2014) and rice (Nakamura 2015b; Nishi et al. 2001; Tetlow and Emes 2014). The high amylose and branch chain length distribution are key influencers of starch digestibility (Tetlow and Emes 2014). The production of resistant starches in sorghum is poorly understood (Griebel et al. 2019).

The relative amounts and structural characteristics of amylose and amylopectin are under genetic control (Tetlow et al. 2004). The starch-synthesis pathway (SSP) is a conserved pathway across cereal species (Tetlow et al. 2004). Located in the endosperm of the seed, adenosine 5' diphosphate glucose pyrophosphorylase (AGPase), granule bound starch synthases (GBSSs), starch synthases (SSs), starch branching enzymes (SBEs) and two types of debranching enzymes, isoamylases and pullulanases, are the key groups of enzymes involved in starch biosynthesis (Tetlow et al. 2004). The different enzymes vary in their catalytic activities (Guan and Preiss 1993; Nakamura 2015a; Tetlow et al. 2004; Tetlow and Emes 2014). Hill et al. (2012) reported that mutations in SSIIa and SBEIIb result in increased amylose values in sorghum. Amylose extender is regulated by SBEIIb (Jane 2009; Nakamura 2015b; Nishi et al. 2001; Tetlow and Emes 2014). The Su2 gene codes for SSIIa in corn (Liu et al. 2012b; Zhang et al. 2004) and contribute to low starch GT (Liu et al. 2012b; Preiss 2009; Shannon et al. 2009; Zhang et al. 2004). High amylose in sorghum was associated with higher starch GT (Hill et al. 2012). The starch GT phenotype in rice is reported to be controlled by the SSSII-3 (ALK) gene encoding a starch synthase II (Gao et al. 2011), also controlling the ASV phenotype (Gao et al. 2011; Tian et al. 2009). In sorghum, the genes controlling the ASV phenotype and how ASV relates to starch characteristics is poorly understood.

A rapid assay to measure alkali spreading value (ASV) is commonly used in rice breeding to select for genotypes with varying starch GT (Little et al. 1958). The ASV phenotype is negatively correlated with starch GT in rice (Bhattacharya 1979; Bhattacharya and Sowbhagya 1972; Juliano et al. 1964; Mariotti et al. 2010; Tan and Corke 2002). Griebel et al. (2019) adapted the ASV test used in rice with a scale of 0-7 (Little et al. 1958) into a binary scale for use in sorghum to identify genotypes with modified starch properties. Mutants with an ASV+ phenotype exhibited lower to higher starch GTs compared to BTx623; thus, providing new opportunities to develop sorghum varieties with modified starch GT (Griebel et al. 2019). The ASV assay is of great benefit to preselect for varying starch GT genotypes but cannot replace DSC to differentiate lower and higher starch GT (Griebel et al. 2019). So far, it is poorly understood how the ASV phenotype of sorghum relates to different starch characteristics such as amylose content, resistant starch and paste viscosity profiles.

Given reports in rice, the initial hypothesis was that a SSIIa gene, involved in starch biosynthesis, controls the ASV phenotype in sorghum seed endosperm. However, many genes and enzymes are known to be involved in starch 
biosynthesis and processing and these genes may also contribute to variation in ASV. In this study, sorghum mutants (EMS ethyl methane sulfonate) exhibiting an ASV+ phenotype were used to identify the genes controlling the alkali spreading phenotype of sorghum based on whole-genome re-sequencing and genetic segregation analyses. Genotypes with single or double mutations were created to assess starch properties including amylose content, starch GT and paste viscosity profiles. Grain samples produced from these mutants were also characterized for variation in starch quality and related food-processing parameters. Genes, alleles and SNP markers for use in breeding sorghum varieties with varying starch GT and composition are reported.

\section{Materials and methods}

\section{Plant material}

A sorghum EMS population was developed using BTx623 (Krothapalli et al. 2013; Addo-Quaye et al. 2018). The ASV test was used in a forward genetic screen to identify Sorghum bicolor EMS (SbEMS) mutants with altered starch phenotypes (Griebel et al. 2019). Seed of nine SbEMS mutant lines exhibiting altered ASV phenotype were produced in Puerto Rico during the 2016-2017 production season (PR16/17). These lines and controls BTx623, Macia and Sepon82 were also grown in West Lafayette 2017 (WL17) and 2018 (WL18). The trials were grown in West Lafayette under rainfed conditions with long days. The trials in Puerto Rico were grown under short day conditions with irrigation. Two biological replicates per genotype and year except three replicates of BTx623 in WL18 were tested for ASV, amylose content and paste viscosity profiles.

To conduct an allele dosage test, A-lines for each mutant were developed by crossing each mutant line as a pollen donor to ATx623 followed by backcrossing to the $F_{1}$ over several generations. The mutant $\mathrm{B}$-lines were used as the recurrent parents in each generation. The mutant B-lines are registered as genetic stocks in the USDA National Plant Germplasm System at the National Laboratory for Genetic Resources Preservation (PI 690479 SbEMS 2703; PI 690480 SbEMS 2773, PI 690481 SbEMS 3194, PI 690482 SbEMS 3218, PI 690483 SbEMS 3403, PI 690484 SbEMS 3568, PI 690485 SbEMS 3920, PI 690486 SbEMS 4308, PI 690487 SbEMS 5890).

Double mutants were created by hand emasculating and crossing selected EMS mutants in 2017 (SbEMS $3194 \times$ SbEMS 4308; SbEMS $2703 \times$ SbEMS 4308). The harvested $F_{1}$ seeds were grown in the greenhouse and genotyped for both parental alleles. The verified $F_{1}$ plants were self-pollinated, and the $F_{2}$ populations were grown in a greenhouse and genotyped to identify homozygous double mutant $F_{2}$ plants. The homozygous double mutants were transplanted in the field in 2018, self-pollinated and harvested as single panicles. The parental genotypes were also grown in the field in 2018. The seeds of double mutant lines and two biological replicates for each of the parent lines were subjected to further phenotypic analyses.

\section{DNA preparation}

DNA was extracted from the SbEMS mutants exhibiting an ASV+ phenotype (Griebel et al. 2019) using a modified CTAB extraction protocol (Murray and Thompson 1980). Tissue samples were ground in extraction buffer solution (1.2 M NaCl, $100 \mathrm{mM}$ Tris pH 8, 20 mM EDTA pH8, 2\% CTAB, $0.1 \%$ BME) followed by extraction with chloroform (24 parts chloroform, 1 part isoamyl alcohol). The aqueous layer was transferred to a new Eppendorf tube and diluted with $1070 \mu \mathrm{L}$ dilution buffer $(100 \mathrm{mM}$ Tris $\mathrm{pH} 8,20 \mathrm{mM}$ EDTA $\mathrm{pH} 8,2 \%$ CTAB) followed by precipitation with $530 \mu \mathrm{L}$ wash buffer ( 3 parts TE buffer, 7 parts ethanol). The samples were incubated for $15 \mathrm{~min}$ at room temperature, centrifuged at $12,000(3220 \times g)$ for $10 \mathrm{~min}$, and the pellet was re-suspended in $53 \mu \mathrm{L}$ high-salt TE $[1 \mathrm{M} \mathrm{NaCl}, 10 \mathrm{mM}$ Tris pH 8, 2 mM EDTA pH 8, RNaseA $(50 \mu \mathrm{g} / \mathrm{mL})]$. The samples were incubated at $60{ }^{\circ} \mathrm{C}$ for $15 \mathrm{~min}$ and then diluted with $212 \mu \mathrm{L} \mathrm{H}_{2} \mathrm{O}$. DNA was precipitated with $530 \mu \mathrm{L}$ pure $\mathrm{EtOH}$ at $-80{ }^{\circ} \mathrm{C}$ for $30 \mathrm{~min}$, centrifuged $(3220 \times g)$ for $10 \mathrm{~min}$, and the pellet was washed with $70 \%$ ethanol. The pellets were re-suspended in TE buffer ( $10 \mathrm{mM}$ Tris $\mathrm{pH} 8$, 1 mM EDTA pH 8).

The fast DNA extraction method described by Xin et al. (2003) was used for DNA extractions for co-segregation analyses.

\section{Whole-genome re-sequencing and analyses}

Whole-genome re-sequencing data for all the sorghum samples were generated at the Purdue Genomic Core Facility. One sorghum mutant, SbEMS 3218, had undergone next-generation sequencing as described by Addo-Quaye et al. (2018), and SNP data were reported at the Functional Gene Discovery Platform for Sorghum (https://www.purdu e.edu/sorghumgenomics/). The other eight EMS mutants SbEMS 2703, SbEMS 2773, SbEMS 3194, SbEMS 3403, SbEMS3568, SbEMS 3920, SbEMS4308 and SbEMS 5890 were processed as described below. The library construction was conducted using the TruSeq ${ }^{\circledR}$ DNA PCR-Free Sample Preparation Guide from Illumina. The samples SbEMS 2773, SbEMS 3194, SbEMS 3568 and SbEMS 5890 were PCR amplified as the final library concentration was too low after the PCR-free protocol step. The samples were sequenced using Illumina HiSeq 2500 with 100 bp pairedend reads. The filtered NGS read files (R1 and R2) obtained 
from the Purdue Genomics Core were cleaned of contaminants by aligning the R1 and R2 files to the indexed Phix 174 genome using Bowtie 2 short reads aligner (Langmead and Salzberg 2012). Next, NGS reads with origins in the sorghum mitochondrial genome were filtered by using Bowtie 2 to detect alignment to the mitochondrial genome $\mathrm{NC}$ 008360.1 downloaded and freely available from the National Center for Biotechnology Information (NCBI) sequence read archive at https://www.ncbi.nlm.nih.gov/sra.

A reference genome-based read mapping approach was used for SNP calling and quality filtering. The Sorghum bicolor reference genome BTx623 (Paterson et al. 2009) assembly and annotation sequences (version 3.0; Sbicolor_313_v3.1) were downloaded from Phytozome database version 11 (Goodstein et al. 2012). The reference genome assembly sequences were indexed using Bowtie2 build. The filtered NGS paired-end reads were aligned to the indexed sorghum reference genome using Bowtie 2 (Langmead and Salzberg 2012). The reference genome sequences were indexed separately using the faidx and CreateSequenceDictionary programs in the SAMtools alignment manipulation software (Li et al. 2009) and the GATK genome analysis toolkit packages (McKenna et al. 2010; DePristo et al. 2011), respectively. The tool GATK was used for SNP calling (McKenna et al. 2010; DePristo et al. 2011). The SAM-formatted output files containing the aligned sorghum NGS reads were sorted and indexed using the sort and index programs in the SAMtools software package. NGS duplicate reads were tagged using the MarkDuplicates tool in the Picard Tools package (Picard Toolkit 2016). Variant detection was performed in the sorted BAM files using the GATK HaplotypeCaller tool (DePristo et al. 2011; McKenna et al. 2010). The SNP data were submitted as.vcf files to the European Variation Archive (EVA) and can be found under the EVA project PRJEB33472 named "Sorghum EMS mutants exhibit ASV and special starch quality traits." The SNP data are also made available via PURR - the Purdue University Research Repository, and can be found at https://purr.purdu e.edu/publications/3238/1.

\section{Functional annotations of SNP variation}

The effects of identified SNPs on the sorghum gene function were found using snpEff variant effect annotation tool (Cingolani et al. 2012). The default snpEff configuration file was updated with a new database name for the sorghum genome. The Sorghum bicolor reference genome sequences (FASTAformatted file) and the genome annotation information ( $g f f 3$ file) obtained from Phytozome database version 11 (Goodstein et al. 2012) were used to create a new database using snpEff build-gff3 command. Then .vcf files that contain the GATK Haplotype Caller variants were used as input files for the next step. In this next step, a snpEff-annotated variant call .vcf output file containing the variant annotations was created.

\section{Designation of starch-synthesis genes (candidate gene approach)}

A list of candidate genes involved in sorghum starch biosynthesis in the amyloplast was made to check for SNPs in all the genes associated with starch biosynthesis. The majority of genes involved in the amyloplast were obtained from Campbell et al. (2016). The genes Sobic.007G101500 and Sobic.009G127500 were also added to this list based on recent studies (Goodstein et al. 2012, database version 11).

\section{Comparative genomics: multiple sequence alignment}

The protein sequences from Sobic.004G163700 and Sobic.010G093400 were downloaded from Phytozome. The sequence was blasted against all green plants, viridiplantae, in NCBI using tBLASTn (Altschul et al. 1990; Camacho et al. 2009). More and less related species were selected to be able to see whether the detected SNPs are in conserved protein regions across more related species or even across all species. The protein sequences were then downloaded in fasta format. Multiple sequence alignment was conducted using Clustal Omega (Chojnacki et al. 2017), and alignment results were imported into the ClustalW2 package Simple Phylogeny for calculation of distances by the unweighted pair group method algorithm (UPGMA) method. The alignment and clustering packages are freely available from https ://www.ebi.ac.uk/Tools/.

\section{Primerdesign, PCR and SNP genotyping (CAPS, dCAPS, tetra-primer)}

CAPS (Cleaved Amplified Polymorphic Sequences) markers were created for the mutant alleles sbeIIb-2773-2, sbeIIb-5890-7 and sbeIIb-3568-6. The sequence around the SNP was obtained from Phytozome. The software dCAPS Finder 2.0 (Neff et al. 2002) is freely available at http://helix .wustl.edu/dcaps/ and was used with 0 mismatches to find restriction enzymes cutting both forward and reverse strand and only the mutant or wild-type, not both. NCBI PrimerBLAST (Altschul et al. 1990; Camacho et al. 2009) was used to create primers and is freely available from https://www. ncbi.nlm.nih.gov/tools/.

dCAPS (Derived Cleaved Amplified Polymorphic Sequences) were created for the mutant allele sbeIIb-3194-3. The sequence around the SNP was obtained from Phytozome. The dCAPS Finder 2.0 (Neff et al. 2002) was used to create dCAPS with maximum of 2 mismatches. 
Tetra-primer for ARMS-PCR were created for the mutant alleles ssIIa-4308-2, sbeIIb-2703-1, sbeIIb-3403-5 and sbeIIb-3218-4. The sequence around the SNP was obtained from the reference genome. The software PRIMER1: primer design for tetra-primer ARMS-PCR, freely available at http://primer1.soton.ac.uk/prime r1.html, was used to create tetra-primer (Collins and $\mathrm{Ke}$ 2012; Ye et al. 2001).

Primers used for PCR amplification are described in Table 1. The PCR reactions were adjusted to primer-specific annealing temperatures for each mutant. The ratio and amount of primers used were adjusted for tetra-primer types.

Table 1 PCR primers, primer sequences and restriction enzymes used for the genotyping of sorghum EMS mutants

\begin{tabular}{|c|c|c|c|c|c|}
\hline PCR primer ID/type & Gene/SNP position/allele & Primer sequence & Temp. $\left({ }^{\circ} \mathrm{C}\right)$ & Enzyme cut & Product size \\
\hline $\begin{array}{l}2703-3 \_ \\
\text {S12_51294121 } \\
\text { Tetra-primer }\end{array}$ & $\begin{array}{l}\text { Sobic. } 004 G 163700 \\
51294121 \\
\text { sbeIIb-2703-1 }\end{array}$ & $\begin{array}{l}\text { InF: TTGCATTGCCTG } \\
\text { ATCAAACTCGTA } \\
\text { InR: CGGACTATCTTA } \\
\text { GGTATCGTGGTA } \\
\text { GGC } \\
\text { OuF: GACATTACAAGA } \\
\text { AGAATCCCCACCAA } \\
\text { OuR: GTTTGGTCAATA } \\
\text { ATTGATCATTGTCGG }\end{array}$ & 65.2 & None & $\begin{array}{l}\text { Product size } \\
\text { T allele: } 110 \\
\text { C allele: } 165 \\
\text { outer primers: } 224\end{array}$ \\
\hline $\begin{array}{l}2773 \_ \\
\text {04_SNP_51302688 } \\
\text { CAPS }\end{array}$ & $\begin{array}{l}\text { Sobic. } 004 G 163700 \\
51302688 \\
\text { sbeIIb-2773-2 }\end{array}$ & $\begin{array}{l}\text { F: CACGGTAAAGAG } \\
\text { TACCTGCGA } \\
\text { R: TGAGCATGAAGG } \\
\text { AGGCTTGG }\end{array}$ & 57.1 & $\begin{array}{l}\text { ApoI-HF } \\
\text { cuts Mutant (M) }\end{array}$ & $\sim 78(\mathrm{cut} \sim 28, \sim 50 \mathrm{bp})$ \\
\hline $\begin{array}{l}\text { 3194_ } \\
\text { 04_SNP_51298115 } \\
\text { dCAPS }\end{array}$ & $\begin{array}{l}\text { Sobic. } 004 G 163700 \\
51298115 \\
\text { sbeIIb-3194-3 }\end{array}$ & $\begin{array}{c}\text { F: ATCATGGAGGTC } \\
\text { ACACCATCAATGC } \\
\text { R: TCCAATGCTAGA } \\
\text { TGGTGGCTTGAG }\end{array}$ & 59.1 & $\begin{array}{l}\text { HpyCH4 V } \\
\text { cuts M }\end{array}$ & $\sim 71($ cut $\sim 26, \sim 45)$ \\
\hline $\begin{array}{l}3218 \\
\text { S_TET } \\
\text { Tetra-primer }\end{array}$ & $\begin{array}{l}\text { Sobic. } 004 G 163700 \\
51295327 \\
\text { sbeIIb-3218-4 }\end{array}$ & $\begin{array}{l}\text { InF: GTGTGTGCACAA } \\
\text { TATCACCCATCGTT } \\
\text { InR: CAGGCAAAGTGA } \\
\text { TGAAGCTGGG } \\
\text { OuF: ACCTTGTCCATC } \\
\text { AACCAAAATGCA } \\
\text { OuR: CGTGTGCGTTCA } \\
\text { CTTTGAGCTATG }\end{array}$ & 61.0 & None & $\begin{array}{l}\text { Product size } \\
\text { G allele: } 150 \\
\text { A allele: } 100 \text { outer primers: } \\
202\end{array}$ \\
\hline $\begin{array}{l}3403(1) \\
\text { Tetra-primer }\end{array}$ & $\begin{array}{l}\text { Sobic. } 004 G 163700 \\
51295906 \\
\text { sbeIIb-3403-5 }\end{array}$ & $\begin{array}{l}\text { InF: AGTTCAATCCAT } \\
\text { TTGTCAGCCACATCT } \\
\text { InR: GTAGGTTTTGAC } \\
\text { TATCGGATGCACCTG } \\
\text { OuF:CAGCCCATGCAA } \\
\text { TTAAACATTAGT } \\
\text { GTATG } \\
\text { OuR: CCTCCACATCAT } \\
\text { TGGCTTACATAAACC }\end{array}$ & 60.0 & None & $\begin{array}{l}\text { Product size } \\
\text { A allele: } 201 \\
\text { G allele: } 182 \\
\text { outer primers: } 329\end{array}$ \\
\hline $\begin{array}{l}3568 \_ \\
\text {04_SNP_51292780 } \\
\text { CAPS }\end{array}$ & $\begin{array}{l}\text { Sobic. } 004 G 163700 \\
51292780 \\
\text { sbeIIb-3568-6 }\end{array}$ & $\begin{array}{l}\text { F: ACATCTGTGTAC } \\
\text { CAAAGGCGAT } \\
\text { R: CAGGATCCATCA } \\
\text { CGCAGCA }\end{array}$ & 57.2 & $\begin{array}{l}\text { HpyCH4III } \\
\text { cuts WT }\end{array}$ & $\sim 79($ cut $\sim 37, \sim 42)$ \\
\hline $\begin{array}{l}4308 \_ \\
\text {S13_8302675 } \\
\text { Tetra-primer }\end{array}$ & $\begin{array}{l}\text { Sobic.010G093400 } \\
8302675 \\
\text { ssIIa-4308-2 }\end{array}$ & $\begin{array}{l}\text { InF: TTGTTCTGCAAG } \\
\text { GTTGCTGGTA } \\
\text { InR: TGACAGTATAGT } \\
\text { TCAGGGGGATAC } \\
\text { ATC } \\
\text { OuF: TGATGCACCTCT } \\
\text { CTTCCGG } \\
\text { OuR: TTCGCTAGTGCA } \\
\text { AAAGTTGATCC }\end{array}$ & 61.6 & None & $\begin{array}{l}\text { Product size } \\
\text { A allele: } 181 \\
\text { G allele: } 224 \\
\text { two outer primers: } 356\end{array}$ \\
\hline $\begin{array}{l}5890 \_ \\
\text {04_SNP_51301340 } \\
\text { CAPS }\end{array}$ & $\begin{array}{l}\text { Sobic. } 004 G 163700 \\
51301340 \\
\text { sbeIIb-5890-7 }\end{array}$ & $\begin{array}{l}\text { F: TGCTAGCCCAAA } \\
\text { GTAGGAACAA } \\
\text { R: CAGGCTCCAGGA } \\
\text { GAAATACCA }\end{array}$ & 56.4 & MboI cut WT 1x, M 2x & $\begin{array}{l}\sim 121 \text { (cut: } W: \sim 78,43 ; M: \\
\quad \sim 67,11,43 \text { ) }\end{array}$ \\
\hline
\end{tabular}


Polyvinylpyrrolidone (PVP) 20\% and bovine serum albumin (BSA) were added to improve PCR amplification of DNA from fast and dirty extractions (Xin et al. 2003).

Each PCR product from CAPS and dCAPS was digested with enzymes from New England Biolabs under recommended conditions. The allele sbeIIb-3568-6 (3568_04_SNP_51292780) was digested with HpyCH4III, sbelIb-2773-2 (2773_04_SNP_51302688) with ApoI-HF, sbeIIb-5890-7 (5890_04_SNP_51301340) with MboI and sbeIIb-3194-3 (3194_04_SNP_51298115) with CviRI=HpyCH4 V. All samples from enzyme digest or tetraprimers were examined in 2.5-3\% TAE high-resolution agarose gels, depending on the fragment size.

\section{Co-segregation analyses}

The SbEMS mutants were crossed to BTx623 (except SbEMS3920 was un-successful in crossing). $F_{1}$ plants were selected and self-pollinated to create bi-parental $F_{2}$ populations. Panicles of $F_{2}$ plants were self-pollinated, and $F_{2: 3}$ seeds were screened for ASV with 32 seeds per panicle and in rare cases less if limited by seed number. The number of panicles per population screened varied from 30 for SbEMS3403 to 112 for SbEMS2773. $F_{3}$ plants of those $F_{2: 3}$ panicles were grown on a bench containing sand, and leaf tissues were sampled and genotyped. Each panicles' ASV phenotype and the corresponding SNP genotype ( $M=$ mutant, $H=$ heterozygous, $W=$ wild-type) was determined. A Chi-square test was conducted to determine goodness of fit for the 3:1 phenotypic ratio as well as the 1:2:1 genotypic ratio.

\section{Allele dosage test}

An allele dosage test was conducted to determine whether the mutant alleles influenced amylose content in an alleledependent manner. The A-lines and B-lines for each mutant were intercrossed to produce the hybrids for testing inheritance in the triploid endosperm. To obtain zero doses of the mutant allele, BTx623 was used as pollen donator and crossed to ATx623. A single dosage of the mutant allele was created by crossing the mutants onto ATx623. Two dosages of the mutant allele were created by crossing BTx623 onto the A-line (or hand emasculated panicle) of each EMS mutant. Three doses were created by self-pollinating the B-line (West Lafayette 2017) or by crossing the B-line to the corresponding A-line (West Lafayette 2018). The hybrid seeds were analyzed for ASV and amylose content. The experiment was set up to obtain two biological replicates per genotype and allele dosage, but depended on crossing success and seed set in the field. In 2017, the A-line for SbEMS2703 was not available so hybrids were made by hand emasculation. The SbEMS3920 mutant was not included as no co-segregation data were available at that point.

\section{Phenotypic analyses}

The alkali spreading test was conducted as described in Griebel et al. (2019) with $1.8 \% \mathrm{KOH}$ for $24 \mathrm{~h}$ and scored binary as ASV+ or ASV- (wild-type). Thirty-two seeds were randomly chosen and tested. In rare cases, fewer seeds were tested if seed quantity was limited. The randomly chosen seeds for the allele dosage test were cut horizontally so that only the triploid endosperm was evaluated for ASV with 32 seeds per panicle.

The flour preparation was conducted as follows. The seed samples from PR16/17, WL17 and WL18 described above were milled into a fine flour using a ball mill (Retsch, Haan, Germany) as described in Griebel et al. (2019).

The starch extraction and thermal properties were conducted as previously described from flour samples of double mutants and parents (Griebel et al. 2019; Benmoussa and Hamaker 2011). The flour samples of double mutants and parents were analyzed in duplicate for their starch thermal properties using differential scanning calorimetry (DSC) as described by Griebel et al. (2019). The starch thermal properties tested were onset $\left(T_{\mathrm{o}}\right)$, peak $\left(T_{\mathrm{p}}\right)$, conclusion $\left(T_{\mathrm{c}}\right)$ starch GT, range of starch GT $\left(T_{\mathrm{c}}-T_{\mathrm{o}}\right)$ and enthalpy.

The paste viscosity analysis was conducted, and corresponding samples were adjusted based on moisture content. Moisture was determined using the standard oven method (Bradley Jr. 2014). Aluminum pans were oven-dried before use. For moisture determination, $1 \mathrm{~g}$ of flour sample was weighed on pre-weighed aluminum pans and dried over night at $105{ }^{\circ} \mathrm{C}$. In rare cases, flour was limited and less than $1 \mathrm{~g}$ was sampled. The samples were placed in a desiccator after drying and before the final weight was recorded. The weights before and after drying were recorded, and moisture was calculated based on water loss. Paste viscosity analysis was performed on whole grain flour samples using the Rapid Visco Analyzer (RTE-100), model 4 (Serial Number 970835) from Newport Scientific, Australia. The RVA standard method 1 was used, and the results were analyzed using standard analysis 1 . Water-flourdispersions of $11.86 \%$ were prepared (Bouvier and Campanella 2014). For the SbEMS mutants, two biological replicates from WL17 and WL18 were analyzed. Each biological replicate was sampled in duplicate. The double mutants from WL18 did not have enough flour sample, so in some cases the biological replicate was not analyzed in duplicate.

Amylose and amylopectin analyses were performed on whole grain flour samples by following the Megazyme K-AMYL kit manual. Each flour sample from PR16/17, WL17 and WL18 was analyzed in duplicate. The 
spectrophotometer (Spectronic Genesys 10 Bio, Thermo Electron Corporation, Model: 970S0008) was used at $510 \mathrm{~nm}$.

\section{Statistical analyses}

Statistical analyses were performed using SAS version 9.4. (The data analysis for this paper was generated using SAS software, Version 9.4 of the SAS System for Windows. Copyright (C) 2002-2012. SAS Institute Inc. SAS and all other SAS Institute Inc. product or service names are registered trademarks or trademarks of SAS Institute Inc., Cary, NC, USA.) The GLIMMIX procedure was used to perform a linear mixed model analysis of variance (ANOVA) with the Tukey-Kramer multiple comparison adjustment to compare starch GT, amylose and viscosity properties across genotypes and environments.

\section{Results}

\section{Whole-genome re-sequencing of ASV mutants reveals SNPs in starch biosynthesis genes}

Whole-genome re-sequencing studies characterizing the sorghum mutants described by Griebel et al. (2019) revealed homozygous SNPs in two (Sobic.004G163700, Sobic.010G093400) of 19 genes associated with starch biosynthesis (Fig. 1). SbEMS 2703, SbEMS 2773, SbEMS 3194, SbEMS 3218, SbEMS 3403, SbEMS 3568, SbEMS 5890 exhibited homozygous SNPs in Sobic.004G163700, described as a 1-4-alpha glucan starch branching enzyme II (Goodstein et al. 2012, database version 11; Campbell et al. 2016). The mutant alleles of this gene were described as sbeIIb-2703-1, sbeIIb-2773-2, sbeIIb-3194-3, sbeIIb-3218-4, sbeIIb-3403-5, sbeIIb-3568-6 and sbeIIb-5890-7, respectively. SbEMS 3920 and SbEMS 4308 exhibited homozygous SNPs in Sobic.010G093400, described as a starch synthase zSTSII-1 (Campbell et al. 2016). The mutant alleles of this gene were described as ssIIa-39201 and ssIIa-4308-2, respectively. The mutant SbEMS 3568 has a second homozygous SNP in the gene ADP (Sobic.007G101500), which was not further evaluated as it is an Intron-SNP and the ASV phenotype co-segregated with an exon-SNP in Sobic.004G163700 for that mutant. Similarly, SbEMS 5890 has a second homozygous SNP in a starch synthase gene (Sobic.010G093400); predicted to be a synonymous variant and was, therefore, excluded from further consideration.

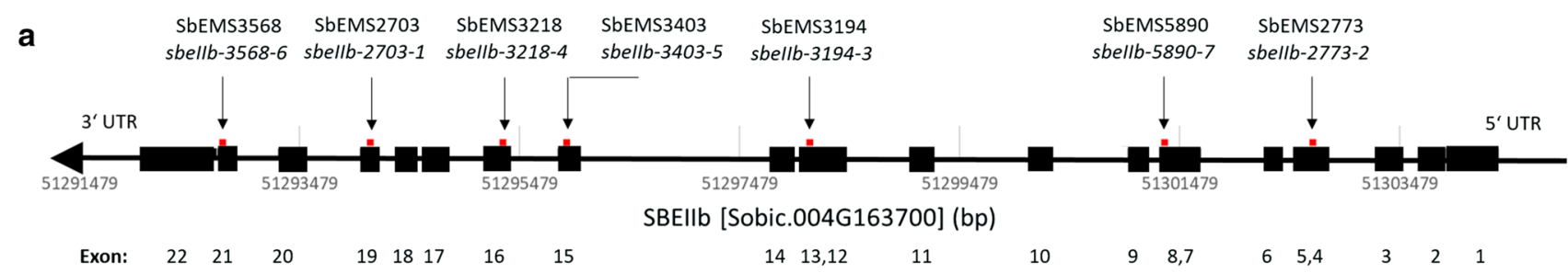

b

\begin{tabular}{|c|c|c|c|c|c|c|c|}
\hline SbEMS & Gene & Allele & \#CHROM & Effect & Impact & Codon Change & Amino Acid Change \\
\hline 2703 & Sobic.004G163700 & sbellb-2703-1 & Chr04 & STOP_GAINED & $\mathrm{HIGH}$ & c. $2053 C>T$ & p.Q685* \\
\hline 2773 & Sobic.004G163700 & sbellb-2773-2 & ChrO4 & MISSENSE_VARIANT & MODERATE & c. $512 \mathrm{G}>\mathrm{A}$ & p.G171E \\
\hline 3194 & Sobic.004G163700 & sbellb-3194-3 & Chr04 & MISSENSE_VARIANT & MODERATE & c. $1345 C>T$ & p.R449C \\
\hline 3218 & Sobic.004G163700 & sbellb-3218-4 & ChrO4 & STOP_GAINED & HIGH & $\operatorname{tgG} / \operatorname{tg} A$ & p.W548* \\
\hline 3403 & Sobic.004G163700 & sbellb-3403-5 & ChrO4 & MISSENSE_VARIANT & MODERATE & c. $1593 \mathrm{G}>\mathrm{A}$ & p.M531I \\
\hline 3568 & Sobic.004G163700 & sbellb-3568-6 & Chr04 & SPLICE_DONOR \& INTRON_VARIANT & HIGH & c. $2328+1 G>A$ & \\
\hline 5890 & Sobic.004G163700 & sbellb-5890-7 & Chr04 & SPLICE_DONOR \& INTRON_VARIANT & HIGH & $c .825+1 G>A$ & \\
\hline
\end{tabular}

C
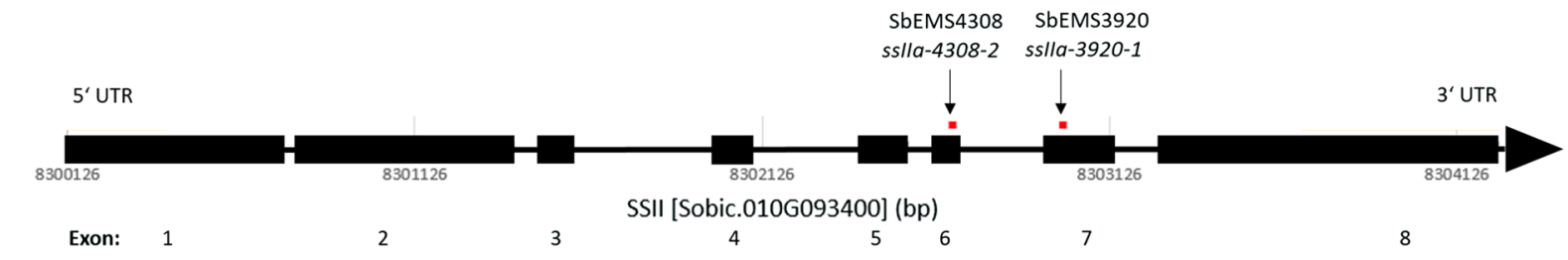

d

\begin{tabular}{|c|c|c|c|c|c|c|c|}
\hline SbEMS & Gene & Allele & \#CHROM & Effect & Impact & Codon Change & Amino Acid Change \\
\hline 3920 & Sobic.010G093400 & ss/la-3920-1 & Chr10 & MISSENSE_VARIANT & MODERATE & c. $1222 \mathrm{G}>\mathrm{A}$ & p.D408N \\
\hline 4308 & Sobic.010G093400 & sslla-4308-2 & Chr10 & MISSENSE \&SPLICE_REGION_VARIANT & MODERATE & c. $1180 \mathrm{G}>\mathrm{A}$ & p.E394K \\
\hline
\end{tabular}

Fig. 1 Mutations in SBEIIb and SSIIa a mutants and positions of SNPs in sbelIb (Sobic.004G163700), b SBEIIb mutations, effects and impacts predicted by snpEff on transcription and translation, $\mathbf{c}$ mutants and positions of SNPs in ssIIa (Sobic.010G093400), and d ssIIa mutations, effects and impacts predicted by snpEff on transcription and translation 
The snpEff tool (Cingolani et al. 2012) was used for functional SNP annotations to predict the effects of each SNP on transcription and translation of Sobic.004G163700 and Sobic.010G09340 (Fig. 1b, d). In Sobic.004G163700, the SNPs within the alleles sbeIIb-3568-6 and sbeIIb-5890-7 are predicted to alter splice donor sites and sbeIIb-2703-1 and sbeIIb-3218-4 created premature stop codons. Moderate protein effects in the form of missense variants (amino acid changes) are predicted for the SNPs in the alleles of sbeIIb-2773-2, sbeIIb-3403-5 and sbeIIb-3194-3. In Sobic.010G093400, the SNP in the allele ssIIa-3920-1 is predicted as a missense variant and the SNP in the allele ssIIa4308-2 is predicted as a missense and splice region variant.

\section{Comparative genomics identifies SSIIa and SBEIIb}

Multiple sequence alignment and BLASTP (Altschul et al. 1990; Camacho et al. 2009) showed that Sobic.004G163700 is likely the previously reported sorghum SBEIIb (AY304540) (Mutisya et al. 2003; De Alencar Figueiredo et al. 2008) as both show $98.9 \%$ sequence similarity (Goodstein et al. 2012, database version 12). Comparisons of the protein sequences and a phylogram (Chojnacki et al. 2017) of Sobic.004G163700 showed that the new alleles are predicted to affect regions of the protein highly conserved across species like Zea mays, Oryza sativa, Hordeum vulgare, Triticum aestivum, Lens culinaris, Pisum sativum, Phaseolus vulgaris and Colocasia esculenta (Fig. 2). The similarity of the SBEIIb in sorghum, followed by maize, rice (sbe3), barley and wheat suggests that the identified gene is a starch branching enzyme of the isoform SBEIIb. This suggested that Sobic.004G163700 encodes a SBEIIb.

Multiple sequence comparison of protein sequences (Chojnacki et al. 2017) similar to that encoded by Sobic.010G093400 showed that the SNPs of ssIIa-3920-1 and ssIIa-4308-2 are in regions highly conserved across species of Glycine max, Triticum aestivum, T. monococcum and T. urartu, Hordeum vulgare, Zea mays, Oryza sativa and Amaranthus cruentus (Fig. 3). The phylogenetic tree showed the sorghum Sobic.010G93400 is most closely related to a sorghum SSIIa followed by SSS2-3 from maize and SSIIa from Triticum.

\section{ASV phenotype co-segregates with SNPs in SSIIa and SBEIIb}

Eight of the SbEMS mutants were crossed to BTx623 and evaluated in $F_{2: 3}$ populations for their ASV phenotype and a

b

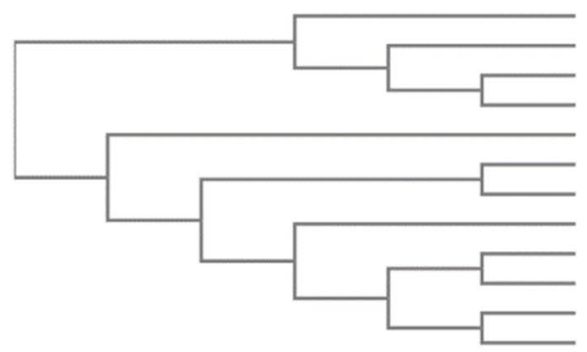

ColocasiaEsculenta.SBEA 0.15813

PhaseolusVulgaris.Sbe2 0.088214

P.Sativum.Sbel 0.0225012

LensCulinaris.Sbell 0.0225012

ZeaMays.Sbella 0.1174

TriticumAestivum.Sbellb 0.0282882

HordeumVulgare.Sbellb 0.0282882

OryzaSativa.Sbe3 0.0940367

ZeaMays.Ae1 0.00377454

ZeaMays.Sbellb 0.00377454

Sobic. 004 G163700 0.00564833

Sorghum.SBEIIb.AY304540 0.00564833

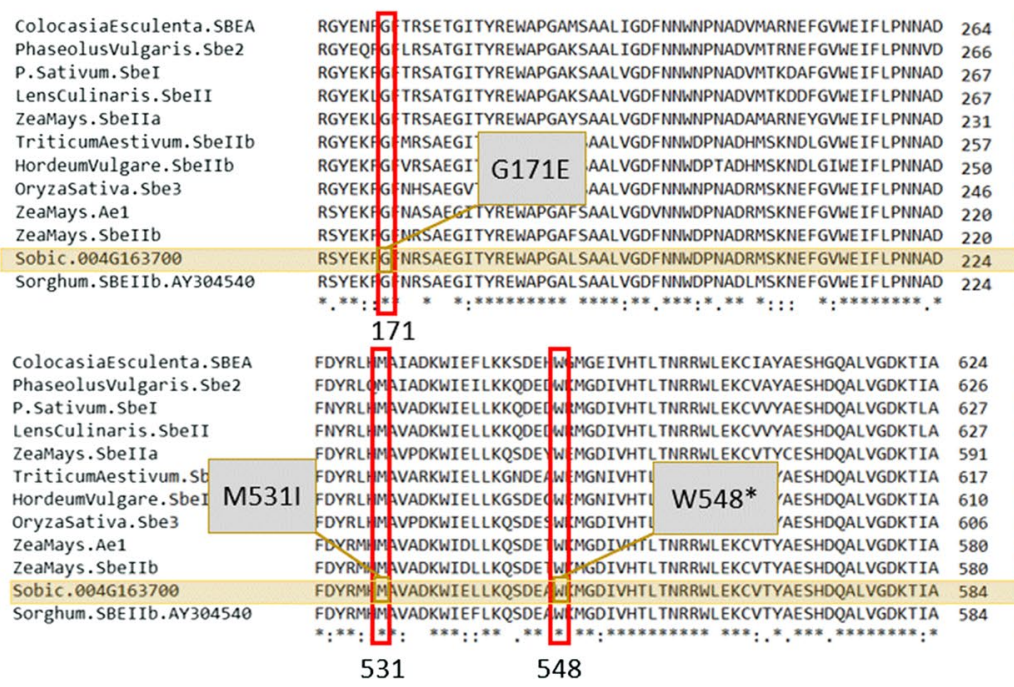

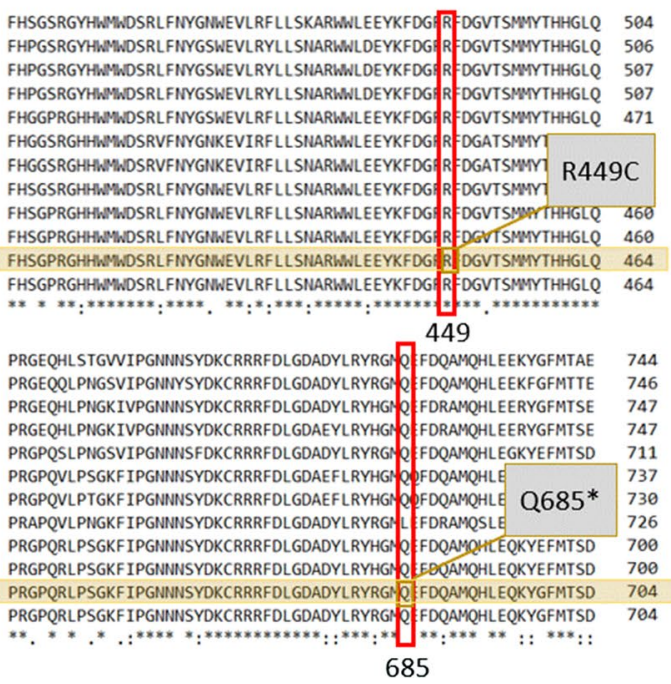

685

Fig. 2 Multiple sequence alignment of protein sequences from different sources with sorghum starch branching enzyme: a phylogram using UPGMA and distance correction b SNPs in regions of the Sobic.004G163700 protein compared to other species 
associated SNP genotype (Fig. 4). Phenotypic evaluations and Chi-square tests provided evidence that the mutant alleles sbeIIb-2773-2, sbeIIb-3218-4, sbeIIb-3403-5 and ssIIa-4308-2 segregated for ASV in a 3:1 ratio (Fig. 4a). The 3:1 ratio did not fit (Chi-square $p$ value) for the alleles sbeIIb-2703-1, sbeIIb-3194-3, sbeIIb-3568-6 and sbeIIb-5890-7. Genotyping studies showed that the sbeIIb-2703-1, sbeIIb-2773-2, sbeIIb-3218-4 and ssIIa4308-2 alleles segregated in a 1:2:1 genotypic ratio but sbeIIb-3194-3, sbeIIb-3403-5, sbeIIb-3568-6 and sbeIIb-5890-7 alleles exhibited distorted ratios (Fig. 4b). This may be due to low viability of the seeds for homozygous mutants. A larger sample size might have fit the 3:1 and $1: 2: 1$ ratios better.
Linkage analyses demonstrated perfect co-segregation between the putative causal SNP markers and the ASV mutant phenotype in bi-parental populations of BTx623 with SbEMS 2703, SbEMS 3218, SbEMS 3403, SbEMS 3568, SbEMS 4308 and SbEMS 5890 (Fig. 4). Genetic analyses in populations developed using SbEMS 2773 and SbEMS 3194 also exhibited strong linkage between the SNP markers and the ASV phenotype. But three progenies for the variant sbeIIb-2773-2 and two progenies for sbeIIb-3194-3 exhibited a panicle with a heterogenous ASV phenotype but leaf tissue of a wild-type genotype. a

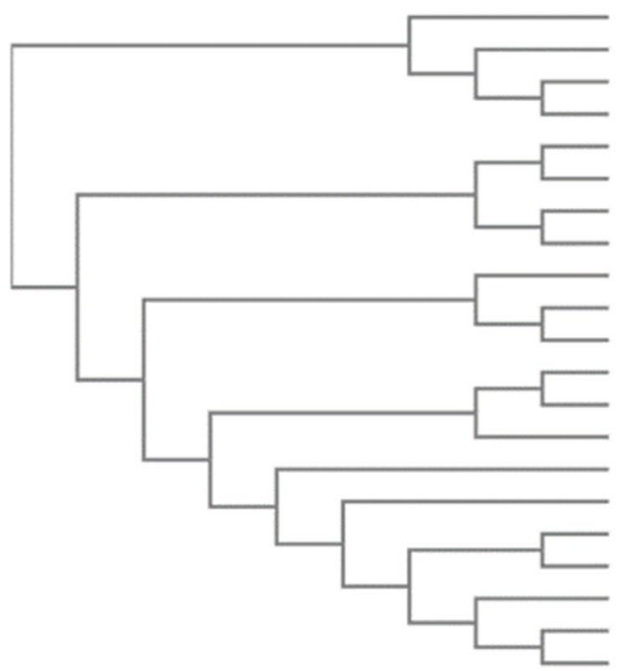

AmaranthusCruentus.SSII 0.241138

Manihot.Esculentas.SSII 0.209056

GlycineMaxSSIla 0.0347782

GlycineMax.SSIla-1 0.0347782

TriticumAestivum.SSIlb-precursor 0.117943

OryzaSativa.SS2 0.117943

SorghumBicolor.SSIlb-precursor 0.0495276

ZeaMays.SSIlb-precursor 0.0495276

OryzaSativalndica.SSIla 0.00185667

OryzaSativa.SSSII-3 0

OryzaSativaJaponicaSSIla 0

Sobic.010G093400.1 0.00202457

SorghumBicolor.SSIla-precursor 0.00202457

ZeaMays.SSS2-3 0.0325941

Hordeum.VulgareSubsp.Vulgare.SSII 0.0423759

TriticumAestivum.SSIla-3 0.0238332

TriticumMonococcumSubspAegilopoides.SSIla 0.00125376

TriticumMonococcumSubspMonococcum.SSIla 0.00125376

TriticumAestivum.SSIIA 0.00314324

TriticumAestivum.SSIla-2 0.00125376

TriticumUrartu.SSIla 0.00125376
Amaranthuscruentus.SSII

TriticumAestivum.SSIIb-precursor oryzasativa.ss2

SorghumBicolor.SSIIb-precursor

ZeaMays.SSIIb-precursor

oryzasativaIndica.SSIIa

Oryzasativa.SSSII-3

OryzasativaJaponicassIIa

Sobic.010G093400.1

SorghumBicolor.SSIIa-precursor

ZeaMays.SSS2-3

Hordeum.vulgaresubsp.vulgare.SSII

TriticumAestivum.SSIIa-3

TriticumMonococcumsubspAegilopoides.SSIIa

TriticummonococcumSubspMonococcum.SSIIa

TriticumAestivum.SSIIA

TriticumAestivum.SSIIa-2

Triticumurartu.ssira

Manihot.Esculentas.SSII

GlycinemaxSSIIa

GlycineMax.SSIIa-1
ILFCKVA E PWHVPCGGVCYCDNLVFIANDWHTALLPVYKAYYRDNGLMKYTRSVLV ILFCKAAYE PRYALCGGTIYCD INLVF IANDWHTALLPVYLKAYYRDNGLMQYTRSVLV ILFCKAAY E PWFAPCGGSIY D PNLVF IANDWHTALLPVYLKAYYRDNGLMQYTRSVLV ILFCKAAYE PWYAPCGGTVY DONLVFIANI IYYRDSGLMQYARSVLV E394K ILFCKAAYE PWYAPCGGTVYDDNLVFIAN D408N IYRDNGLMQYARSVLV ILFCKAAYE PWHVPCGGVPYDDNLVFLANI IYRDNGMMQYTRSVLV TLECKAAY E PWHVPCGGVPYCD FNLVELANDWHTALLPVYLKAYYRDNGMMQYTRSVLV ILFCKAANEVPWHPCGGVPYCDNLVFLANDWHTALLPVYKAYYRDNGMMQYTRSVLV ILFCKVAV E PWHVPCGGVCYCD | FNLVF IANDWHTALLPVYKAYYRDHGLMQYTRSILV ILFCKVAVEYPWHVPCGGVCY DDNLVFIANDWHTALLPVYLKAYYTDHGLMQYTRSILV ILFCKVAY E PWHVPCGGVCYCD PNLVFIANDWHTALLPVYLAYYRDHGLMQYTRSVLV ILFCKAAY E PWHVPCGGVPY D ENLVF IANDWHTAL LPVLKAYYRDHGLMQYSRSVMV ILFCKAAY E PWHVPCGGVPYCDNLVFIANDWHTALLPVYLAYYRDHGLMQYTRSIMV ILFCKAAY E PWHVPCGGVPYCD NLVF IANDWHTALLPVYKAYYRDHGLMQYTRSIMV ILFCKAA E PWHVPCGGVPY D FNLVF IANDWHTALLPVYKAYYRDHGLMQYTRSIMV ILFCKAAY E PWHVPCGGVPY D DNLVFIANDWHTALLPVYLKAYYRDHGLMQYTRSIMV ILFCKAAYE PWHVPCGGVPYCD NLVF IANDWHTALLPVYLKAYYRDHGLMQYTRSIMV ILFCKAAY E PWHVPCGGVPY D NLVF IANDWHTAL LPVIKAYYRDHGLMQYTRSIMV VLFCKAAYE PWHVPCGGVCY D FNLAFIANDWHTALLPVYLKAYYRDNGLMQYTRSVLV VLFCKAAA E PWHVPCGGVCYCD DNLAF IANDWHTALLPVYKAYYRDHGIMKYTRSVLV VLFCKAAAE PWHVPCGGVCYCD INLAF IANDWHTALLPVYLKAYYRDHGLMKYTRSVLV $:^{* * * *}, * \ldots * \ldots, * * * \quad * * * * *,{ }^{*}: * * * * * * * * * * * * * * * * * * *:^{*}:^{*}:^{* *}::^{*}$ $394 \quad 408$
484

Fig. 3 Multiple sequence alignment of protein sequences from different sources with sorghum starch synthase enzyme: a phylogram using UPGMA and distance correction b SNPs in protein regions of Sobic.010G093400 compared to other species 


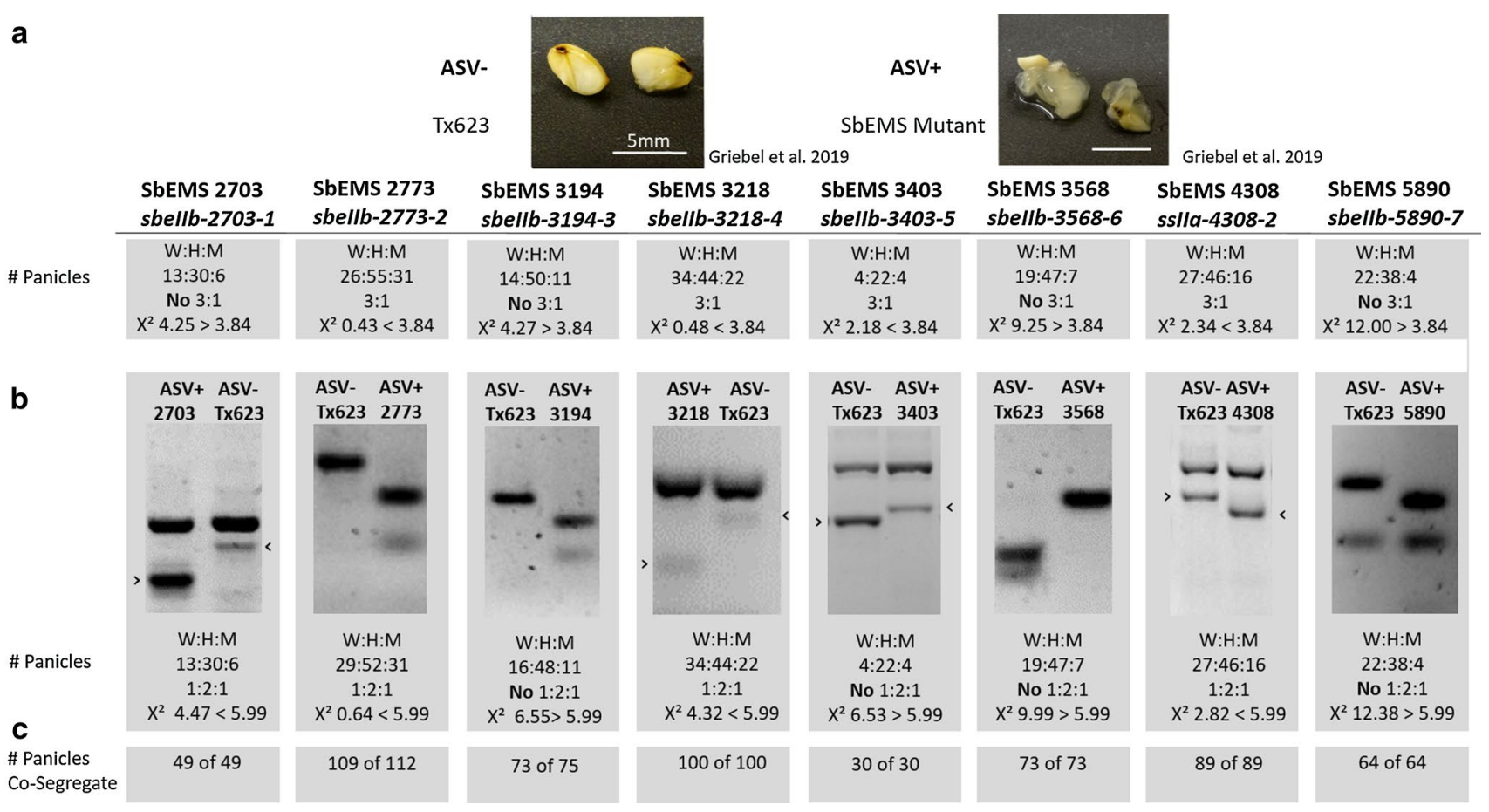

Fig. 4 Co-segregation analyses-a of contrasting ASV phenotypes $(1.8 \% \mathrm{KOH}, 24 \mathrm{~h})$, ASV segregation ratios for each SbEMS mutant, and statistical tests for segregation of ASV, $\mathbf{b}$ SNP markers for each SbEMS mutant, genotype segregation ratios and statistical tests for
SNP segregation, and c co-segregation of SNP markers and ASV. Arrows $(>)$ on gel pictures indicate the inner primers' band of the tetra-primers used

\section{SSIla and SBEIlb mutations result in different starch GT patterns}

The mutants SbEMS 3920 and SbEMS 4308 showed significantly lower starch GT compared to the controls and the other mutants (Griebel et al. 2019). In the current study, it was determined that these genotypes have mutations in Sobic.010G093400 as represented by ssIIa-3920-1 and ssIIa-4308-2. SbEMS 2703, SbEMS 2773, SbEMS 3194, SbEMS 3218, SbEMS 3403, SbEMS 3568 and SbEMS 5890 exhibited the highest starch GT values, approximately $5{ }^{\circ} \mathrm{C}$ higher $T_{\mathrm{p}}$ than the control samples (Griebel et al. 2019). These genotypes were identified as having mutations in Sobic.004G163700 as represented by sbeIIb-2703-1, sbeIIb-2773-2, sbeIIb-3194-3, sbeIIb-3218-4, sbeIIb-3403-5, sbeIIb-3568-6 and sbeIIb-5890-7.

\section{Mutations in sorghum SBEIlb result in increased amylose values}

Comparative studies showed that Sobic.004G163700 is similar to SBEIIb (Mutisya et al. 2003) in sorghum and closely related to Zea mays amylose extender gene Ael; hence, it was hypothesized that mutants with SNP mutations in this gene could have increased amylose values in comparison with controls and SSIIa variants (Table 2). There was no significant genotype by environment interaction (Table S1) observed across three seasons (Puerto Rico 2016/2017, West Lafayette 2017 and 2018), so amylose values are presented for each environment and in a combined analysis. Macia was not evaluated in Puerto Rico 2016/17 but exhibited amylose values similar to the other controls in the other environments.

The sbeIIb mutants sbeIIb-2703-1, sbeIIb-2773-2, sbeIIb-3194-3, sbeIIb-3218-4, sbeIIb-3403-5, sbeIIb-3568-6 and sbeIIb-5890-7 produced significantly higher amylose values (>30\% amylose) compared to the ssIIa mutants ssIIa-3920-1 and ssIIa-4308-2 and the controls. In all three seasons, SbEMS 3194 had the highest amylose values. The SbEMS 2703, SbEMS 3218 and SbEMS 5890 were ranked 2 nd to 4 th in two environments, respectively. The SSIIa mutations represented by ssIIa-3920-1 and ssIIa-4308-2 produced similar amylose values and did not show higher amylose values compared to the controls. 
Table 2 Amylose values for Sorghum bicolor EMS mutants and controls from Puerto Rico 2016/2017 (PR16/17), West Lafayette 2017 (WL17) and West Lafayette 2018 (WL18)

\begin{tabular}{|c|c|c|c|c|c|c|c|c|}
\hline Pedigree & Allele & \multicolumn{4}{|c|}{ Amylose\%-LSMeans } & $\begin{array}{l}\text { PR 16/17 } \\
\text { Rank }\end{array}$ & WL 2017 & WL 2018 \\
\hline EMS2703 & sbeIIb-2703-1 & $43.54 \mathrm{C}$ & $43.60 \mathrm{BC}$ & $38.14 \mathrm{BC}$ & $41.76 \mathrm{D}$ & 2 & 2 & 3 \\
\hline EMS2773 & sbeIIb-2773-2 & $38.16 \mathrm{C}$ & $37.89 \mathrm{BC}$ & $35.79 \mathrm{BC}$ & $37.28 \mathrm{C}$ & 5 & 6 & 6 \\
\hline EMS3194 & sbeIIb-3194-3 & $44.02 \mathrm{C}$ & $48.15 \mathrm{C}$ & $45.10 \mathrm{C}$ & $45.76 \mathrm{E}$ & 1 & 1 & 1 \\
\hline EMS3218 & sbeIIb-3218-4 & $41.40 \mathrm{C}$ & $40.54 \mathrm{BC}$ & $38.18 \mathrm{BC}$ & $40.04 \mathrm{CD}$ & 3 & 3 & 2 \\
\hline EMS3403 & sbeIIb-3403-5 & $36.70 \mathrm{BC}$ & $34.63 \mathrm{AB}$ & $29.26 \mathrm{AB}$ & $33.53 \mathrm{~B}$ & 6 & 7 & 7 \\
\hline EMS3568 & sbeIIb-3568-6 & $36.41 \mathrm{BC}$ & $38.45 \mathrm{BC}$ & $37.94 \mathrm{BC}$ & $37.6 \mathrm{C}$ & 7 & 5 & 4 \\
\hline EMS5890 & sbeIIb-5890-7 & $39.88 \mathrm{C}$ & $40.15 \mathrm{BC}$ & $36.42 \mathrm{BC}$ & $38.82 \mathrm{CD}$ & 4 & 4 & 5 \\
\hline EMS3920 & ssIIa-3920-1 & $26.20 \mathrm{~A}$ & $24.20 \mathrm{~A}$ & $24.76 \mathrm{~A}$ & $25.06 \mathrm{~A}$ & 10 & 12 & 8 \\
\hline EMS4308 & ssIIa-4308-2 & $28.36 \mathrm{AB}$ & $25.41 \mathrm{~A}$ & $23.27 \mathrm{~A}$ & $25.68 \mathrm{~A}$ & 8 & 10 & 11 \\
\hline Macia & control & NA & $27.15 \mathrm{~A}$ & $23.28 \mathrm{~A}$ & NA & & 8 & 10 \\
\hline Sepon82 & control & $24.63 \mathrm{~A}$ & $26.05 \mathrm{~A}$ & $22.71 \mathrm{~A}$ & $24.46 \mathrm{~A}$ & 11 & 9 & 12 \\
\hline Тх623 & control & $26.90 \mathrm{AB}$ & $24.47 \mathrm{~A}$ & $23.94 \mathrm{~A}$ & $25.11 \mathrm{~A}$ & 9 & 11 & 9 \\
\hline
\end{tabular}

Macia not evaluated in season Puerto Rico 16/17, so not part of combined analysis. Values followed by the same letter (A-E) in the same column are not significantly different

\section{Mutations in SBEIIb alter amylose values and ASV phenotypes}

Based on the higher amylose values of sbeIIb-2703-1, sbeIIb-2773-2, sbeIIb-3194-3, sbeIIb-3218-4, sbeIIb-3403-5, sbeIIb-3568-6 and sbeIIb-5890-7 allelic variants (Table 2) and sequence similarity to ae of maize, it was proposed that Sobic.004G163700 could be a sorghum homolog of amylose extender. Based on this proposal, the amylose level in sorghum may depend on the number of alleles in the triploid endosperm. To test this hypothesis, each of the sbeIIb mutants was sterilized in A1-cytoplasm and the A-lines and B-lines of mutants and Tx623 were used in crosses to produce seeds carrying zero, one, two and three mutant alleles in the triploid endosperm. The amylose values increased in a dosage-dependent and mutant alleledependent manner for sbeIIb mutants and did not increase in ssIIa mutants. In most cases, amylose content increased by $1 \%$ to $2 \%$ with each additional dose of sbeIIb in the endosperm with values greater than $30 \%$ when three mutant sbeIIb alleles were present (Table 3). This shift in amylose content was not observed in samples with varying doses of ssIIa (SbEMS 4308). Analyses of samples for ASV in 2018 showed an increase in percentage of seeds with an ASV phenotype depending on whether one, two or three mutant $s b e I I b$ or ssIIa alleles were present in the endosperm. No ASV phenotype was observed in genotypes carrying one dose of the allele.

\section{The sbellb and sslla alleles show distinct paste viscosity profiles}

Pasting viscosity is an important property of starch related to its gelatinization. The paste viscosity profiles are very distinct for ssIIa and sbeIIb mutants in both environments. There was a significant genotype by environment effect for the pasting viscosity profiles (Table S2); therefore, data are presented by individual environment (Fig. 5). The sbeIIb mutants exhibited lower peak and final viscosity compared to ssIIa-4308-2, ssIIa-3920-1 and controls (Fig. 5a, b). The viscosity profiles of BTx623, Sepon82, Macia, ssIIa3920-1 and ssIIa-4308-2 followed the same trend in both environments. The sbeIIb mutants slightly overlap but cluster together across environments. The SNP mutations in Sobic.010G093400, encoding SSIIa, resulted in reduced viscosity in comparison with the controls but higher viscosity in comparison with sbeIIb mutants. BTx623 showed an earlier and larger breakdown than all other starches (Fig. 5a-c). The mutant starches demonstrated minimal breakdown and later peak viscosity or a plateau from peak viscosity. The setback viscosity, formation of a gel as the long chain amylose molecules begin to realign, was $\sim 10 \times$ lower in the sbeIIb mutants compared to wild-type starches.

\section{Double mutants of SSIIa and SBEIIb resemble the SBEIIb parent in phenotype}

SbEMS mutant B-lines were hand emasculated and intercrossed to develop the $F_{2}$ populations sbeIIb-3194-3 $\times$ ssIIa4308-2 and sbeIIb-2703-1 × ssIIa-4308-2 (Fig. 6). Homozygous double mutants were identified in each population. 


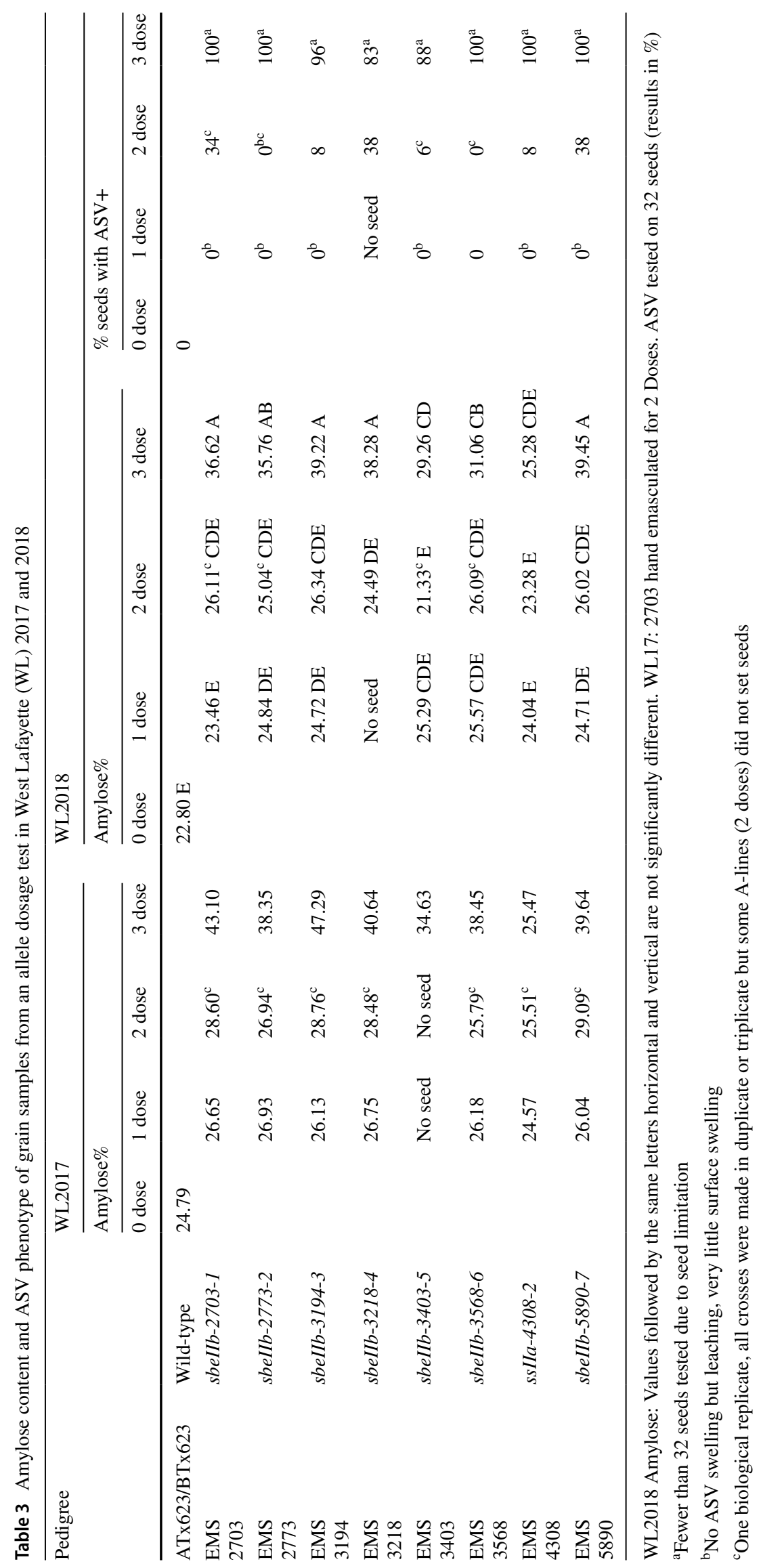




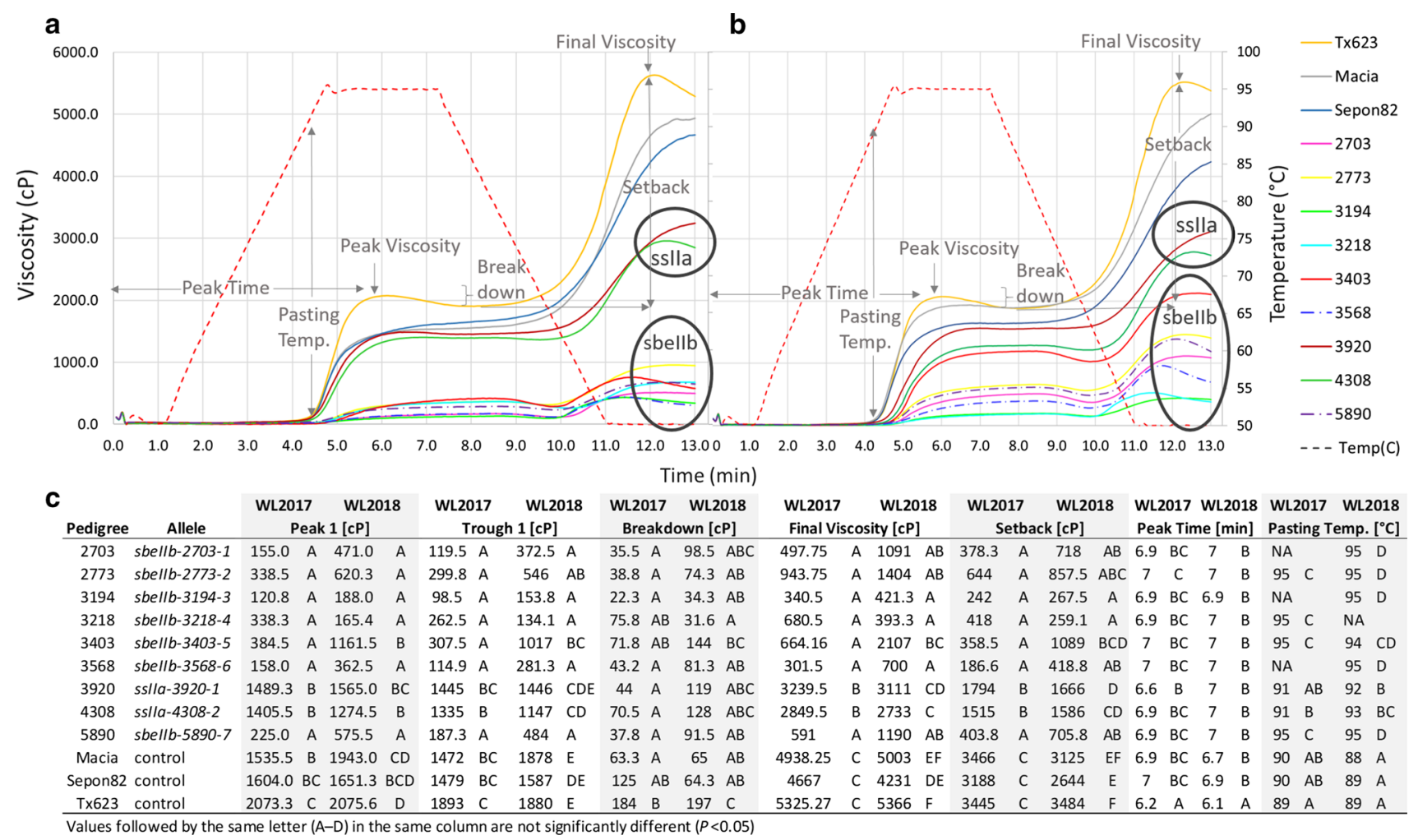

Fig. 5 RVA paste viscosity profiles of sorghum EMS mutants and controls-lsmean values presented from two seasons a West Lafayette (WL) 2017 and $\mathbf{b} 2018$ and $\mathbf{c}$ both seasons. Two biological replicates per genotype and three for BTx623 in WL2018

Each double mutant has one parent with the ssIIa-4308-2 allele and a second parent with either the sbeIIb-2703-1 or sbeIIb-3194-3 alleles. The lowest amylose values were measured in the parent carrying ssIIa-4308-2 with $23 \%$ and higher amylose values in parent sbeIIb-2703-1 with 38\% and sbeIIb-3194-3 with 44\%. The amylose values in the double mutants resembled the sbeIIb mutant parent and ranged from 37 to $39 \%$. The lowest starch GT values were measured in the parent carrying ssIIa-4308-2 and the higher values in parent sbeIIb-2703-1 and sbeIIb-3194-3. The starch thermal properties of the double mutants also resembled the sbeIIb mutant parent. The paste viscosity profiles varied depending on the mutant alleles involved and resulted in higher peak and final viscosity in ssIIa-4308-2 than in sbeIIb-2703-1 or sbeIIb-3194-3. The viscosity profiles for the double mutants were similar to their sbeIIb mutant parents.

\section{Discussion}

Whole-genome re-sequencing of ASV mutants in sorghum revealed SNPs in starch biosynthesis genes. Seven new alleles of a starch branching enzyme (Sobic.004G163700) and two new alleles of a starch synthase (Sobic.010G093400) were identified in this study. Comparative genomics provided evidence that Sobic.010G93400 encodes a SSIIa. Sobic.010G093400 is most similar to SSSII-3 in rice and SSIIa in sorghum and other crop species. Sequence comparisons suggest that Sobic.004G163700 codes for a SBEIIb with high sequence similarity to the one reported previously by Mutisya et al. (2003) and is closely related to amylose extender (SBEIIb) in maize (Fig. 2). The SBEIIb of sorghum reported by Mutisya et al. (2003) showed high sequence similarity with other cereals too. The protein sequence obtained from NCBI of maize amylose extender and sorghum SBEIIb, AY304540, were BLAST against sorghum in the database Phytozome (Goodstein et al. 2012). The BLAST search exhibited the best hit for Sobic.010G273800, followed by Sobic.003G213800 and Sobic.004G163700. All three genes are reported as similar to branching enzymes (Campbell et al. 2016), while Sobic.003G213800 is listed as a putative 1-4-alpha glucan branching enzyme, Sobic.010G273800 as similar to a starch branching enzyme I precursor, and Sobic.004G163700 is similar to a 1-4-alpha glucan branching enzyme 2 (Campbell et al. 2016). No SNPs were found in Sobic.010G273800 or Sobic.003G213800 of ASV mutants. The gene Sobic.004G163700 appears to be a sorghum homolog of the maize gene amylose extenderl (ael) encoding a SBEIIb. 


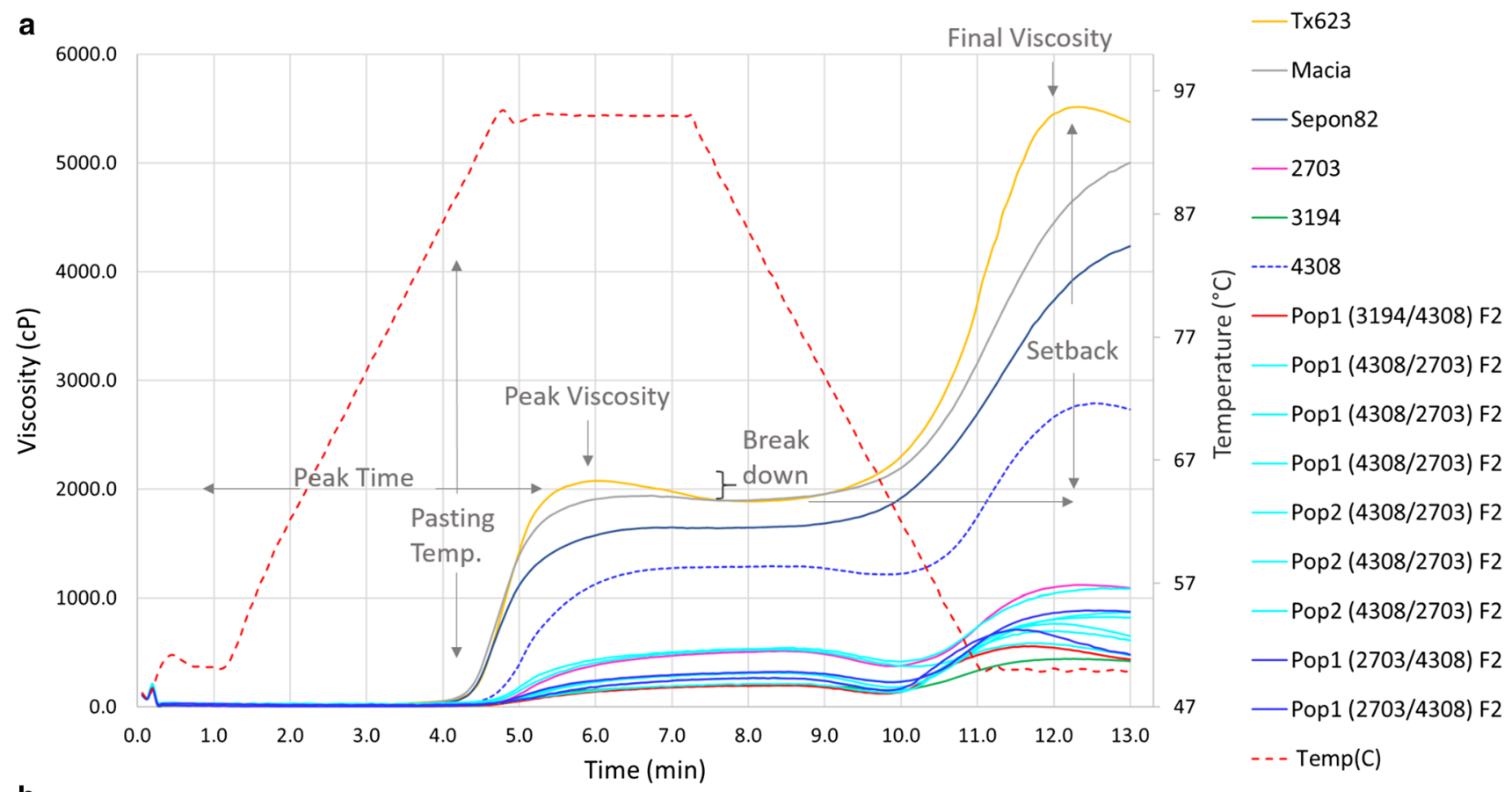

b

\begin{tabular}{|c|c|c|c|c|c|c|c|c|c|}
\hline \multirow{3}{*}{ Pedigree } & \multirow{3}{*}{ Allele } & \multicolumn{3}{|c|}{ Amylose } & \multicolumn{5}{|c|}{ starch gelatinization characteristics } \\
\hline & & \multirow[t]{2}{*}{ Reps } & [\%] & To $\left[{ }^{\circ} \mathrm{C}\right]$ & $\operatorname{Tp}\left[{ }^{\circ} \mathrm{C}\right]$ & $\mathrm{Tc}\left[{ }^{\circ} \mathrm{C}\right]$ & TC-To $\left[{ }^{\circ} \mathrm{C}\right]$ & Enthalpy $[\mathrm{J} / \mathrm{g}]$ & Melting Point $\left[{ }^{\circ} \mathrm{C}\right]$ \\
\hline & & & \multicolumn{7}{|c|}{ Ismeans } \\
\hline 2703 & sbellb-2703-1 & 2 & $37.55 \mathrm{AB}$ & $67.6 \mathrm{~A}$ & $78.3 \mathrm{~A}$ & $91.4 \mathrm{AB}$ & $23.7 \mathrm{~A}$ & $4.3 \mathrm{~A}$ & $99.6 \mathrm{AB}$ \\
\hline 3194 & sbellb-3194-3 & 2 & $44.24 \mathrm{~A}$ & $67.3 \mathrm{~A}$ & $80.0 \mathrm{~A}$ & $97.5 \mathrm{~A}$ & $30.1 \mathrm{~A}$ & $2.5 \mathrm{~A}$ & $100.0 \mathrm{~A}$ \\
\hline 4308 & sslla-4308-2 & 2 & $23.34 \mathrm{~B}$ & $55.7 \mathrm{~B}$ & $63.3 \mathrm{~B}$ & $76.7 \mathrm{~B}$ & $21.0 \mathrm{~A}$ & $4.3 \mathrm{~A}$ & 97.7 B \\
\hline Pop1 (2703/4308) F2 & sbellb-2703-1/ss/la-4308-2 & 2 & $38.82 \mathrm{AB}$ & $65.4 \mathrm{~A}$ & $77.3 \mathrm{~A}$ & $88.8 \mathrm{AB}$ & $23.3 \mathrm{~A}$ & $2.9 \mathrm{~A}$ & $100.2 \mathrm{~A}$ \\
\hline Pop1 (3194/4308) F2 & sbellb-3194-3/ss/la-4308-2 & 1 & $37.65 \mathrm{AB}$ & $65.1 \mathrm{~A}$ & $77.1 \mathrm{~A}$ & $87.8 \mathrm{AB}$ & $22.7 \mathrm{~A}$ & $2.4 \mathrm{~A}$ & $99.7 \mathrm{AB}$ \\
\hline Pop1 (4308/2703) F2 & sslla-4308-2/sbellb-2703-1 & 3 & $39.19 \mathrm{~A}$ & $65.0 \mathrm{~A}$ & $77.6 \mathrm{~A}$ & $88.8 \mathrm{AB}$ & $23.8 \mathrm{~A}$ & $3.0 \mathrm{~A}$ & $99.4 \mathrm{AB}$ \\
\hline Pop2 (4308/2703) F2 & sslla-4308-2/sbellb-2703-1 & 3 & $37.40 \mathrm{AB}$ & $64.2 \mathrm{~A}$ & $76.1 \mathrm{~A}$ & $88.6 \mathrm{AB}$ & $24.4 \mathrm{~A}$ & $3.4 \mathrm{~A}$ & $99.7 \mathrm{AB}$ \\
\hline
\end{tabular}

Values followed by the same letter (A to $B)$ in the same column are not significantly different $(P<0.05)$.

Fig. 6 Double mutants of sbeIIb and ssIIa - a RVA paste viscosity profiles $\mathbf{b}$ starch thermal properties and amylose content. Pop $=F_{2}$ Population, numbers (Pop1, Pop2) indicate different populations. Reps $=$ No. of replications)

The seven new alleles of SBEIIb represent moderate-tohigh-impact SNP mutations spread across the entire gene and may provide a resource for understanding the gene function in sorghum. The central catalytic A-domain of SBEIIb in maize and rice has four highly conserved regions important for the catalytic activity with key amino acid positions and a catalytic triad (Tetlow and Emes 2014). The mutant allele sbeIIb-3194-3 results in the substitution R449C, which is within a catalytic group in SBEIIb of sorghum (Mutisya et al. 2003) and the position Arg445 in maize SBEIIb (Tetlow and Emes 2014; Fig. 2). The amino acid changes in alleles sbeIIb-3403-5 (M531I) and sbeIIb-3218-4 (W548*) might be also in the catalytic domain of SBEIIb, as this is close to a region of importance for catalytic activity in maize SBEIIb (Tetlow and Emes 2014).

None of the nine ASV mutants with new allelic variants of SSII a and SBEIIb have a SNP mutation in the GBSS (Sobic.002G116000, Sobic.010G022600; Campbell et al.
2016), known to act on amylose (Nakamura 2015a). It was therefore concluded that the mutants predominantly affect amylopectin synthesis. The endosperm-specific enzyme, SBEIIb, is reported to transfer shorter chains with a degree of polymerization (DP) of $\sim 6$ to 7 in corn, rice and wheat (Tetlow and Emes 2014; Nakamura 2015a). It is possible that the seven sbeIIb mutant alleles lack the enzyme function or show reduced activity, resulting in amylopectins with less short glucan chains of $\mathrm{DP}<8$ and a less branched molecule. An increase in chains of DP $\geq 12-16$ in amylopectin is associated with increased starch GT (Nakamura 2015b). Therefore, having a lack of short chains might change the ratios within the amylopectin molecule and more intermediate sized chains are present, which may explain the high starch GTs of our sbeIIb mutants. In monocots, SSIIa is involved in the elongation of short chains $(\mathrm{DP}<12)$ into intermediate size chains (DP 12-24) (Tetlow et al. 2004; Nakamura 2015b), explaining why ssII mutants in other species result 
in amylopectin with less intermediate chains and more short chains (Nakamura 2015a). An increase in amylopectin short chains of DP $<12$ and a decrease in chains with DP 13 to 24 decrease starch GT (Nakamura 2015b), which could explain the low starch GT of the ssIIa mutants reported in this study. The sbeIIb mutants reported here might behave like amylose extenders with longer internal branch chains of amylopectin (Jane 2009; Tetlow and Emes 2014; Nishi et al. 2001), less branched in outer chains (Jane 2009; Tetlow and Emes 2014) and have less short chains (Jane 2009; Tetlow and Emes 2014; Nishi et al. 2001).

The ASV in sorghum is not solely controlled by a SSII as reported in previous studies in sorghum and rice (Gao et al. 2011; McKneight 2015; Wang et al. 2007) but instead is controlled by at least two genes, Sobic.004G163700 and Sobic.010G093400. Linkage analysis demonstrated that SNP markers for both genes co-segregated with the ASV mutant phenotype in bi-parental populations. It is possible to conclude that each SNP is either the causal mutation or linked to a gene-causing ASV that is in a larger chromosome block. The co-segregation of seven sbeIIb alleles in Sobic.004G163700 with the ASV phenotype provides strong evidence that this gene is one of the genes controlling ASV in sorghum. Similarly, ssIIa also co-segregated with the ASV phenotype in one population supporting the conclusion that Sobic.010G093400 also controls ASV.

The ssIIa and sbeIIb mutations result in different starch GT patterns. A comparative genomics approach showed that sorghum SSIIa is related to rice SSSII-3. The SSSII-3 (ALK) gene is the major gene that regulates starch GT (starch GT measured by ASV) in rice (Tian et al. 2009). The proposed ssIIa alleles in sorghum reported in the current study lower starch GT by $\sim 10{ }^{\circ} \mathrm{C}$, similar to maize (Zhang et al. 2004; Liu et al. 2012b), while a study of natural variation in ssIIa in sorghum (Hill et al. 2012) showed no such distinct changes in starch GT. The lower starch GT is reported to be related to amylopectin structure of ssII mutants (Preiss 2009). The sbeIIb allelic variants behave differently and confirm earlier reports that higher amylose contents are observed together with an increase in starch GT (starch GT measured by ASV) (Tian et al. 2009). In transgenic rice, the position of mutations in the $A L K$ gene influenced whether ASV scores were correlated with starch GT (Gao et al. 2011). The expression of ASV in sorghum occurs for ssIIa or sbelIb allelic variants, and it was not observed that ASV expression changed for different alleles of a gene.

Amylose extender mutant starches are known in maize and rice (Shannon et al. 2009; Li et al. 2008; Liu et al. 2012a; Nishi et al. 2001). The current study reports that Sobic.004G163700 (SBEIIb) is similar in sequence and function to Ael in maize. The new sorghum sbellb alleles result in varying amylose content with higher values overall in comparison with the ssIIa mutants, which is similar to ae maize and rice starches (Shannon et al. 2009; Li et al. 2008; Nishi et al. 2001). The amylose levels measured were $>30 \%$ in this study while $>50 \%$ in maize ( $\mathrm{Li}$ et al. 2008; Liu et al. 2012a). The amylose levels for the controls were similar to previous reports (Beta et al. 2000; Waniska and Rooney 2000; Sang et al. 2008). Mutations of SBEIIb could be used to produce high-amylose sorghum varieties. Different ae alleles in maize are reported to have different starch thermal properties (Shannon et al. 2009; Li et al. 2008; Liu et al. 2012a) such as observed in the current study with sbeIIb mutants resulting in higher $T_{\mathrm{p}}$ and conclusion starch GT $\left(T_{\mathrm{c}}\right)$ and in a wider starch gelatinization range compared to the wild-type (Griebel et al. 2019). Hill et al. (2012) reported that starch GT increased with increased amylose content in sorghum. The ssII mutants showed higher amylose values in other cereals (Zhang et al. 2004; Liu et al. 2012b; Luo et al. 2015), and nearly no change in amylose content in rice (Luo et al. 2015). No change in amylose content was observed for ssIIa-3920-1 and ssIIa-4308-2 in sorghum, similar to reports in rice (Luo et al. 2015).

The performance of hybrids reported in this study showed that sbeIIb mutations are recessive with an allele dosagedependent influence on the amylose content in the triploid endosperm. These observations are very similar to studies for ae in maize (Shannon et al. 2009; Nishi et al. 2001). The wild-type allele was not completely dominant over the mutant sbeIIb allele as reported for ae in other species (Shannon et al. 2009).

The sbeIIb and ssIIa mutants show distinct paste viscosity profiles. It was observed that sbeIIb sorghum mutants have a low gel consistency. In rice, the gene $W x$ controls amylose content and gel consistency (GC), both traits being negatively correlated (Tian et al. 2009). In rice, amylose content is also regulated by minor genes such as the $A L K$ (SSII-3) gene, SSIII-2, SSI and PUL (Tian et al. 2009). Minor genes controlling GC in rice are ISA, SBE3 (Tian et al. 2009) and the $A L K$ (SSII-3) gene (Gao et al. 2011; Tian et al. 2009). The peak viscosity is related to the maximum swelling capacity of starch granules (BeMiller and Huber 2008; Bouvier and Campanella 2014) and is mostly determined by the amylopectin content of the starch (Bouvier and Campanella 2014). This property is associated with the thickening power of a starch (Biliaderis 2009). The swelling capacity and thickening power of the high amylose sbeIIb mutants in the present study are lower than in controls and ssIIa mutants, which imply that sbeIIb mutants are less suitable to be used as thickening agents. The breakdown is reported to depend on the starch structure with high amylopectin starches breaking down faster (Biliaderis 2009), as observed in the results for BTx623 but not for ssIIa-3920-1 and ssIIa-4308-2. During cooling, the controls, ssIIa-4308-2 and ssIIa-3920-1 formed stronger gels, while the sbeIIb variants were more unstable and sometimes liquid dispersions 
and did not form a proper gel, which is likely related to the extent of molecule retrogradation and re-association influencing gel formation (BeMiller and Huber 2008; Biliaderis 2009; Bouvier and Campanella 2014). Higher amylopectin starches such as those produced by the ssIIa mutants and the controls, resulted in better gel consistency and gel volume development than sbeIIb mutants, as amylopectin and amylose-amylopectin interactions are the important drivers of these properties (Biliaderis 2009).

The ASV mutants fall into distinct classes regarding functional properties and effects on starch thermal characteristics, amylose levels and pasting behavior. The sbeIIb genetic variants exhibited higher $T_{\mathrm{p}}$ and $T_{\mathrm{c}}$, a wider range of starch GT (Griebel et al. 2019), higher amylose levels and lower peak and final viscosity compared to the controls. Peak viscosity was positively correlated with gel consistency and negatively correlated with starch GT as previously shown in rice (Gao et al. 2011). Low amylose content together with high gel consistency and low starch GT (Griebel et al. 2019) were observed in the sorghum ssIIa mutants carrying the alleles ssIIa-3920-1 and ssIIa-4308-2, which are desired cooking qualities in rice (Wang et al. 2007). The ssIIa mutants exhibit a lower peak and final viscosity than the controls but higher peak and final viscosity than the high amylose sbeIIb allelic variants. Hill et al. (2012) reported an increase in amylose content by genetic variants of ssII a and sbeIIb resulting in higher starch GT, higher final viscosity, high setback, low peak viscosity and low breakdown. A clear distinction was observed between ssIIa and sbeIIb genes on amylose content, starch GT and pasting behavior with associated changes in lower final viscosity, small breakdown, smaller setback and lower peak viscosity. These results might be due to the allelic variation represented in natural variation of sorghum as compared to genotypes derived by chemical mutagenesis.

Double mutants of amylose extender and sugary2 (su2) have been reported to exhibit amylose values and starch GT more similar to the amylose extender parent in other crops (Shannon et al. 2009). The sorghum double mutants described in this study also resemble the sbeIIb-2703-1 and sbeIIb-3194-3 parents for amylose content, starch thermal properties and starch viscosity profiles. It is not clear if the sbeIIb influences ssIIa or is dominant over it. The $A L K$ (SSII-3) gene in rice did not contribute strongly to changes in paste viscosity profiles as observed for the $w x$ locus (Wang et al. 2007). Variants of the $w x$ locus were not characterized in our study of sorghum mutants; however, it was observed that the allele ssIIa-4308-3 did not contribute to the paste viscosity profiles of double mutants.

The branch chain length and branching frequency influence starch digestibility (Tetlow and Emes 2014). Therefore, it would be of great importance to further evaluate the newly identified alleles for their branch chain length distribution and branching frequency. Genotypes with an $a e$ mutation and high levels of amylose are reported as being correlated with resistant starch content (Jane 2009; Tetlow and Emes 2014; Li et al. 2008) and are less enzyme-digestible (Tetlow and Emes 2014; Li et al. 2008). It may be valuable to determine whether the sbeIIb mutants produce more resistant or slowly digested starches.

Nine novel alleles of a SBEIIb and SSII $a$ were identified and characterized in a Sorghum bicolor EMS population. These mutants exhibited an ASV phenotype and varying starch functional properties. The ASV phenotype was controlled by SSII $a$ and SBEIIb. The sbeIIb variants resulted in lower paste viscosity profiles, lower gel consistency, lower thickening power, higher amylose levels and higher starch GT (especially $T_{\mathrm{c}}$ ) than wild-type samples or ssIIa mutants. The ssIIa mutant alleles resulted in a drop of final viscosity, less gel consistency, lower thickening power, same amylose values and lower starch GT compared to wild-type samples from Tx623. These mutations provide opportunities to produce sorghum varieties with modified starch end-use qualities likely important for the beer brewing and baking industries and specialty foods for humans with diabetes. The fast and cheap ASV assay adapted to sorghum can be easily implemented as a pre-selection step in plant breeding programs around the globe. However, the ASV assay will not completely replace the use of DSC and RVA technologies and requires those analyses on preselected ASV+ genotypes.

Acknowledgments This research was made possible by the support of the Bill and Melinda Gates Foundation and support of the American people provided to the Feed the Future Innovation Lab for Collaborative Research on Sorghum and Millet through the United States Agency for International Development (USAID). The contents are the sole responsibility of the authors and do not necessarily reflect the views of USAID or the United States Government. Program activities are funded by the United States Agency for International Development (USAID) under Cooperative Agreement No. AID-OAA-A-13-00047.

Author contribution statement SG designed and performed all areas of research, SC helped on amylose analysis, AA helped with genotyping, SG analyzed the data, RPW provided support on NGS analysis, CA-Q helped with NGS analysis on a subset of mutants, SG created tables, figures and graphs; $\mathrm{PB}, \mathrm{AA}$ provided technical training, CFW, MRT contributed seeds for the base EMS population, SG wrote the manuscript; coauthors reviewed and edited the manuscript, and CFW, MRT, BAC, OHC guided research, MRT served as the major advisor and project lead.

\section{Compliance with ethical standards}

Conflict of interest The authors declare that they have no conflict of interest.

Open Access This article is distributed under the terms of the Creative Commons Attribution 4.0 International License (http:// 
creativecommons.org/licenses/by/4.0/), which permits unrestricted use, distribution, and reproduction in any medium, provided you give appropriate credit to the original author(s) and the source, provide a link to the Creative Commons license, and indicate if changes were made.

\section{References}

Addo-Quaye C, Tuinstra M, Carraro N, Weil C, Dilkes BP (2018) Whole-genome sequence accuracy is improved by replication in a population of mutagenized sorghum. G3 (Bethesda) 8:1079-1094

Altschul SF, Gish W, Miller W, Myers EW, Lipman DJ (1990) Basic local alignment search tool. J Mol Biol 215:403-410

BeMiller J (2014) Carbohydrate analysis. In: Nielsen S (ed) Food analysis, 4th edn. Springer, New York, pp 147-180

BeMiller JN, Huber KC (2008) Carbohydrates. In: Damodaran S, Parkin K, Fennema O (eds) Fennema's food chemistry, 4th edn. CRC Press, Boca Raton, pp 83-154

Benmoussa M, Hamaker BR (2011) Rapid small-scale starch isolation using a combination of ultrasonic sonication and sucrose density separation. Starch/Stärke 63:333-339

Beta T, Corke H, Rooney LW, Taylor JRN (2000) Starch properties as affected by sorghum grain chemistry. J Sci Food Agric $81: 245-251$

Bhattacharya KR (1979) Gelatinization temperature of rice starch and its determination. In: International Rice Research Institute, proceedings of the workshop on chemical aspects of rice grain quality. International Rice Research Institute, Philippines, pp 231-249

Bhattacharya KR, Sowbhagya CM (1972) An improved alkali reaction test for rice quality. J Food Sci Technol 7:323-331

Biliaderis CG (2009) Structural transitions and related physical properties of starch. In: BeMiller J, Whistler R (eds) Starch chemistry and technology, 3rd edn. Academic Press, Amsterdam, pp 293-372

Bouvier JM, Campanella OH (2014) Quality analysis of extrusiontextured food products. In: Bouvier J-M, Campanella OH (eds) Extrusion processing technology: food and non-food biomaterials. Wiley, Oxford, pp 311-349

Bradley RL Jr (2014) Moisture and total solids analysis. In: Nielsen S (ed) Food analysis, 4th edn. Springer, New York, pp 85-104

Camacho C, Coulouris G, Avagyan V, Ma N, Papadopoulos J, Bealer K, Madden TL (2009) BLAST+: architecture and applications. BMC Bioinformatics 10:421

Campbell BC, Gilding EK, Mace ES, Tai S, Tao Y, Prentis PJ, Thomelin P, Jordan RJ, Godwin ID (2016) Domestication and the storage starch biosynthesis pathway: signatures of selection from a whole sorghum genome sequencing strategy. Plant Biotechnol J 14:2240-2253

Chojnacki S, Cowley A, Lee J, Foix A, Lopez R (2017) Programmatic access to bioinformatics tool from EMBL-EBI update: 2017. Nucleic Acids Res 45:W550-W553

Cingolani P, Platts A, Wang LL, Coon M, Nguyen T, Wang L, Land SJ, Lu X, Ruden DM (2012) A program for annotating and predicting the effects of single nucleotide polymorphisms, SnpEff: SNPs in the genome of Drosophila melanogaster strain $\mathrm{w}^{1118}$; iso-2; iso-3. Fly 6:80-92

Collins A, Ke X (2012) Primer1: primer design web service for tetraprimer ARMS-PCR. Open Bioinform J 6:55-58

De Alencar Figueiredo LF, Calatayud C, Dupuits C, Billot C, Rami J-F, Brunel D, Perrier X, Courtois B, Deu M, Glaszmann M-C (2008) Phylogeographic evidence of crop neodiversity in Sorghum. Genetics 179:997-1008

DePristo MA, Banks E, Poplin R, Garimella KV, Maguire JR, Hartl C, Philippakis AA, del Angel G, Rivas MA, Hanna M, McKenna A, Fennell TJ, Kernytsky AM, Sivachenko AY, Cibulskis K, Gabriel
SB, Altshuler D, Daly MJ (2011) A framework for variation discovery and genotyping using next-generation DNA sequencing data. Nat Genet 43:491-498

Desam GP, Li J, Chen G, Campanella O, Narsimhan G (2018) A mechanistic model for swelling kinetics of waxy maize starch suspension. J Food Eng 222:237-249

Englyst KN, Liu S, Englyst HN (2007) Nutritional characterization and measurement of dietary carbohydrates. Eur J Clin Nutr 61:S19-S39

Food and Agriculture Organization of the United Nations (2019a) Land and water database. FAO, Rome. http://www.fao.org/land-water/ databases-and-software/crop-information/sorghum/en/. Accessed 2019

Food and Agriculture Organization of the United Nations (2019b) FAOSTAT database. FAO, Rome. http://www.fao.org/faostat/ en/. Accessed 2019

Gao Z, Zeng D, Cheng F, Tian Z, Guo L, Su Y, Yan M, Jiang H, Dong G, Huang Y, Han B, Li J, Qian Q (2011) ALK, the key gene for gelatinization temperature, is a modifier gene for gel consistency in rice. J Integr Plant Biol 53:756-765

Goodstein DM, Shu S, Howson R, Neupane R, Hayes RD, Fazo J, Mitros T, Dirks W, Hellsten U, Putman N, Rokhsar DS (2012) Phytozome: a comparative platform for green plant genomics. Nucleic Acids Res 40(Database Issue):178-186. https://doi. org/10.1093/nar/gkr944

Griebel S, Webb MM, Campanella OH, Craig BA, Weil CF, Tuinstra MR (2019) The alkali spreading phenotype in Sorghum bicolor and its relationship to starch gelatinization. J Cereal Sci 86:41-47

Guan HP, Preiss J (1993) Differentiation of the properties of the branching isozymes from maize (Zea Mays). Plant Physiol 102:1269-1273

Hill H, Lee LS, Henry RJ (2012) Variation in sorghum starch synthesis genes associated with differences in starch phenotype. Food Chem 131:175-183

House LR, Gomez M, Murty DS, Sun Y, Verma BN (2000) Development of some agricultural industries in several African and Asian countries. In: Smith CW, Frederiksen RA (eds) Sorghum origin, history, technology and production. Wiley, New York, pp 131-190

Jane J (2009) Structural features of starch granules II. In: BeMiller J, Whistler R (eds) Starch chemistry and technology. Elsevier, Amsterdam, pp 193-236

Juliano BO, Bautista GM, Lugay JC, Reyes AC (1964) Rice quality, studies on the physicochemical properties of rice. J Agric Food Chem 12:131-138

Kimber CT (2000) Origins of domesticated Sorghum and its early diffusion to India and China. In: Smith CW, Frederiksen RA (eds) Sorghum origin, history, technology and production. Wiley, New York, pp 3-98

Krothapalli K, Buescher EM, Li X, Brown E, Chapple C, Dilkes BP, Tuinstra MR (2013) Forward genetics by genome sequencing reveals that rapid cyanide release deters insect herbivory of Sorghum bicolor. Genetics 195:309-318

Langmead B, Salzberg SL (2012) Fast gapped-read alignment with Bowtie 2. Nat Methods 9:357

Li L, Jiang H, Campbell M, Blanco M, Jane J-1 (2008) Characterization of maize amylose-extender (ae) mutant starches. Part I: relationship between resistant starch contents and molecular structures. Carbohydr Polym 74:396-404

Li H, Handsaker B, Wysoker A, Fennell T, Ruan J, Homer N, Durbin $\mathrm{R}$ (2009) The sequence alignment/map format and SAMtools. Bioinformatics 25:2078-2079

Little RR, Hilder GB, Dawson EH (1958) Differential effect of dilute alkali on 25 varieties of milled white rice. Cereal Chem 35:111-126

Liu F, Ahmed Z, Lee EA, Donner E, Lui Q, Ahmed R, Morell MK, Emes MJ, Tetlow IJ (2012a) Allelic variants of the amylose 
extender mutation of maize demonstrate phenotypic variation in starch structure resulting from modified protein-protein interactions. J Exp Bot 63:1167-1183

Liu F, Romanova N, Lee EA, Ahmed R, Evans M, Gilbert EP, Morell MK, Emes MJ, Tetlow IJ (2012b) Glucan affinity of starch synthase IIa determines binding of starch synthase I and starchbranching enzyme IIb to starch granules. Biochem J 448:373-387

Luo J, Ahmed R, Kosar-Hashemi B, Larroque O, Butardo VM Jr, Tanner GJ, Colgrave ML, Upadhyaya NM, Tetlow IJ, Emes MJ, Millar A, Jobling SA, Morell MK, Li Z (2015) The different effects of starch synthase IIa mutations or variation on endosperm amylose content of barley, wheat and rice are determined by the distribution of starch synthase I and starch branching enzyme IIb between the starch granule and amyloplast stroma. Theor Appl Genet 128:1407-1419

Mariotti M, Fongaro L, Catenacci F (2010) Alkali spreading value and image analysis. J Cereal Sci 52:227-235

McKenna A, Hanna M, Banks E, Sivachenko A, Cibulskis K, Kernytsky A, Garimella K, Altshuler D, Gabriel S, Daly M, DePristo MA (2010) The genome analysis toolkit: a MapReduce framework for analyzing next-generation DNA sequencing data. Genome Res 20:1297-1303

McKneight MM (2015) Functional genomics of sorghum grain quality traits. Master thesis, Purdue University

Murray MG, Thompson WF (1980) Rapid isolation of high molecular weight plant DNA. Nucleic Acid Res 8:4321-4325

Mutisya J, Sathish P, Chuanxin S, Andersson L, Ahlansberg S, Baguma Y, Palmqvist S, Odhiambo B, Åman P, Jansson C (2003) Starch branching enzymes in sorghum (Sorghum bicolor) and barley (Hordeum vulgare): comparative analyses of enzyme structure and gene expression. J Plant Physiol 160:921-930

Nakamura Y (2015a) Biosynthesis of reserve starch. In: Nakamura Y (ed) Starch metabolism and structure. Springer, Tokyo, pp 161-209

Nakamura Y (2015b) Manipulation of rice starch properties for application. In: Nakamura Y (ed) Starch metabolism and structure. Springer, Tokyo, pp 335-369

Neff MM, Turk E, Kalishman M (2002) Web-based primer design for single nucleotide polymorphism analysis. Trends Genet 18:613-615

Nishi A, Nakamura Y, Tanaka N, Satoh H (2001) Biochemical and genetic analysis of the effects of amylose-extender mutation in rice endosperm. Plant Physiol 127:459-472

Paterson AH, Bowers JE, Bruggmann R, Dubchak I, Grimwood J, Gundlach H, Haberer G, Hellsten U, Mitros I, Poliakov A, Schmutz J, Spannagl M, Tang H, Wang X, Wicker T, Bharti AK, Chapman J, Feltus FA, Gowik U, Grigoriev IV, Lyons E, Maher CA, Martis M, Narechania A, Otillar RP, Penning BW, Salamov AA, Wang Y, Zhang L, Carpita NC, Freeling M, Gingle AR, Hash CT, Keller B, Klein P, Kresovich S, McCann MC, Ming R, Peterson DG, Rahman M, Ware D, Westhoff P, Mayer KFX, Messing J, Rokhsar DS (2009) The Sorghum bicolor genome and the diversification of grasses. Nature 457:551-556
Picard Toolkit (2016) Broad institute, GitHub repository. http://broad institute.github.io/picard/. Accessed 2016

Preiss J (2009) Biochemistry and molecular biology of starch biosynthesis. In: BeMiller J, Whistler R (eds) Starch chemistry and technology. Elsevier, Amsterdam, pp 83-148

Sang Y, Bean S, Seib PA, Pedersen J, Shi Y-C (2008) Structure and functional properties of sorghum starches differing in amylose content. J Agric Food Chem 56:6680-6685

Shannon JC, Garwood DL, Boyer CD (2009) Genetics and physiology of starch development. In: BeMiller J, Whistler R (eds) Starch chemistry and technology. Elsevier, Amsterdam, pp 23-82

Tan Y, Corke H (2002) Factor analysis of physiochemical properties of 63 rice varieties. J Sci Food Agric 82:745-752

Taylor JRN (1992) Mashing with malted grain sorghum. J Am Soc Brew Chem 50:13-18

Taylor J, Dewar J (2000) Fermented products: beverages and porridges. In: Smith CW, Frederiksen RA (eds) Sorghum origin, history, technology and Production. Wiley, New York, pp 751-796

Tetlow IJ, Emes MJ (2014) A review of starch-branching enzymes and their role in amylopectin biosynthesis. IUBMB Life 66:546-558

Tetlow IJ, Morell MK, Emes MJ (2004) Recent developments in understanding the regulation of starch metabolism in higher plants. $\mathrm{J}$ Exp Bot 55:2131-2145

Tian Z, Qian Q, Liu Q, Yan M, Liu X, Yan C, Liu G, Gao Z, Tang S, Zeng D, Wang Y, Yu J, Gu M, Li J (2009) Allelic diversities in rice starch biosynthesis lead to a diverse array of rice eating and cooking qualities. PNAS 106:21760-21765

Wang LQ, Liu WJ, Xu Y, He XQ, Luo LJ, Xing YZ, Xu CG, Zhang Q (2007) Genetic basis of 17 traits and viscosity parameters characterizing the eating and cooking quality of rice grain. Theor Appl Genet 115:463-476

Waniska RD, Rooney LW (2000) Structure and chemistry of the sorghum caryopsis. In: Smith CW, Frederiksen RA (eds) Sorghum origin, history, technology and production. Wiley, New York, pp 649-688

Xin Z, Velten JP, Oliver MJ, Burke JJ (2003) High-throughput DNA extraction method suitable for PCR. Biotechniques 34:820-826

Ye S, Dhillon S, Ke X, Collins AR, Day IN (2001) An efficient procedure for genotyping single nucleotide polymorphisms. Nucleic Acids Res 29:E88

Zhang X, Colleoni C, Ratushna V, Sirghie-Colleoni M, James MG, Myers AM (2004) Molecular characterization demonstrates that the Zea mays gene sugary2 codes for the starch synthase isoform SSIIa. Plant Mol Biol 54:865-879

Publisher's Note Springer Nature remains neutral with regard to jurisdictional claims in published maps and institutional affiliations. 